\title{
The Spectral Fingerprint of Excited-State Energy Transfer in Dendrimers through Polarization-Sensitive Transient- Absorption Pump-Probe Signals: On-the-Fly Nonadiabatic Dynamics Simulations
}

Deping Hü, Jiawei Peng ${ }^{\dagger \neq}$, Lipeng Chen ${ }^{\#}$, Maxim F. Gelin", Zhenggang Lan ${ }^{\dagger *}{ }^{*}$

${ }^{\dagger}$ SCNU Environmental Research Institute, Guangdong Provincial Key Laboratory of Chemical Pollution and Environmental Safety \& MOE Key Laboratory of Environmental Theoretical Chemistry, South China Normal University, Guangzhou 510006, China

${ }^{t}$ School of Environment, South China Normal University, Guangzhou 510006, China

\#Max Planck Institute for the Physics of Complex Systems, 01187 Dresden, Germany

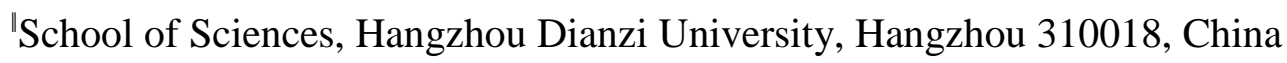

KEYWORDS: transient-absorption pump-probe spectrum, doorway-window representation, onthe-fly, surface hopping, phenylene ethynylene dendrimer 


\section{Abstract}

The time-resolved polarization-sensitive transient-absorption (TA) pump-probe (PP) spectra are simulated using on-the-fly surface-hopping nonadiabatic dynamics and the doorway-window (DW) representation of nonlinear spectroscopy. A typical dendrimer model system composed of two linear phenylene ethynylene units (2-ring, 3-ring) is taken as an example. The fewest switches trajectory surface hopping algorithm along with the TDDFT method is adopted in the nonadiabatic dynamics simulations. The ground-state bleach (GSB), stimulated emission (SE), excited-state absorption (ESA) contributions as well as the total TA PP signals are obtained and carefully analyzed. The correlations between these signals and the coupled nuclear-electronic dynamics are established. It is shown that intramolecular excited-state energy transfer from the 2-ring unit to the 3-ring unit can be conveniently monitored and accurately identified by employing pump and probe pulses with different polarizations. Our on-the-fly nonadiabatic simulation results demonstrate that time-resolved polarization-sensitive TA PP signals provide a powerful tool for the elucidation of excited-state energy transfer pathways, notably in molecular systems possessing several opticallybright nonadiabatically-coupled electronic states with different orientations of transition dipole moments. 
The dendrimer-type compounds display excellent photo-harvesting and exciton-transport properties. As a consequence, they received considerable research interests owing to their potential applications in solar energy conversions. ${ }^{1-7}$ A typical group of dendrimers comprises phenylene ethynylene (PE) branches with different lengths, such as 2-ring, 3-ring and 4-ring linear PE units. ${ }^{8-}$ ${ }^{16}$ Because each branch shows a different length, the electronic excited states of PE-based dendrimers are in principle localized at each PE unit, giving the so-called locally-excited (LE) states. Under radiation fields, the LE states resonant to the radiative frequency are excited, and then the efficient excited-state energy transfer occurs between different units. These photoharvesting and exciton-transfer features were widely studied by both experimental and theoretical works. $^{2-3,8,12-24}$

The experimental studies showed that the introduction of larger conjugated units (for instance, ethynylperylene groups) does not modify the short-wavelength $(<400 \mathrm{~nm})$ bands in the absorption spectra of dendrimer systems, while the emissions of these compounds are fully due to the fluorescence of ethynylperylene groups. ${ }^{6}$ In a word, the short-wavelength absorption relevant to the electronic transition of the short PE units is hardly influenced by the addition of the ethynylperylene groups. However, the efficient excited-state energy transfers must take place among different branches, quenching the emission from the LE state located at the short-length units and enhancing fluorescence emission from the LE state located at the ethynylperylene group. Several time-resolved spectroscopic studies suggested that the ultrafast energy migration takes place from the short-length PE units to the long-length PE units. ${ }^{15,25-26}$ Swallen and coworkers 
provided the upper limits of the energy migration timescales from 4-ring to the core and from periphery to the core, which are $10 \mathrm{ps}$ and $270 \mathrm{ps,} \mathrm{respectively.} \mathrm{Kleiman} \mathrm{and} \mathrm{coworkers} \mathrm{confirmed}$ the existence of LE states and the occurrence of further excited-state energy transfer in conjugated dendrimer systems. ${ }^{15,26}$

From a theoretical perspective, when the same-length PE units are involved in dendrimers, the excited states may become delocalized among several units at the ground-state minimum geometry due to symmetry reasons. ${ }^{21}$ When the PE units have different conjugated lengths, the frontier $\pi$ and $\pi^{*}$ orbitals become localized at corresponding branches, leading to the formation of LE state at each unit. The electronic characters of these LE states were carefully examined by electronic structure calculations and transition density analyses at different levels of theories. ${ }^{19}$ Particularly, the energy of the LE state localized at the short-length unit is generally higher than that at the long-length unit. This gives the unidirectional energy-transfer gradient from the shortlength units to the long-length units. In this sense, such systems represent ideal prototypes in the theoretical study of intramolecular excited-state energy transfer dynamics. The time-resolved spectroscopy provides valuable information to understand such intramolecular excited-state energy transfer, so it should be highly interesting to simulate the time-resolved spectra directly.

Pioneer works by Tretiak, Mukamel and their coworkers give the physical insight into the exciton transfer in PE dendrimers. ${ }^{1}$ For instance, the electronic excitations of dendrimers can be broken into several chromophores, and it should be possible to control the funneling of excitation energy to the desired site by adjusting the lengths of the para-substituted segments in each 
generation. ${ }^{27}$ The interesting interplay exists between the entropic driving force towards the periphery and the energy gradient driving the energy transfer towards the center. ${ }^{17}$ The femtosecond pump-probe (PP) spectra of dendrimer nanostars, which were simulated with the nonlinear exciton equations in conjunction with the doorway-windows (DW) formalism, showed the essential role of exciton coupling in the exciton funneling process. ${ }^{16}$

The excited-state energy transfer processes in PE dendrimers involve both electronic and nuclear motions. The on-the-fly nonadiabatic dynamics simulation is an excellent tool to investigate these problems. Roitberg, Shalashilin, Fernandez-Alberti, Tretiak and coworkers performed a series of on-the-fly nonadiabatic molecular dynamics simulations with surface hopping and multiconfigurational Ehrenfest approaches at the semiempirical AM1/CIS level to study the intramolecular energy transfer of dendrimer systems. ${ }^{2,18,22,28}$ (see also Ref. ${ }^{4}$ for a recent review). Fluorescence anisotropy kinetics were also simulated on-the-fly, though by adopting drastically simplified theoretical assumptions ${ }^{23-24}$. Their works clearly demonstrated that several low-lying excited states mainly correspond to the LE states located at different PE branches, and ultrafast nonadiabatic transitions between different excited states finally result in the efficient intramolecular energy transfer. In their studies, the unidirectional energy transfer dynamics from the short-length conjugated branching subunit to the long-length conjugated branching subunit was clearly addressed. For a typical PE dendrimer example, they showed that the exciton motion from the 2-ring to 3-ring units takes place within $40 \mathrm{fs}$.

If the time-resolved spectra can be simulated by the combination of nonlinear response 
theories and on-the-fly nonadiabatic dynamics, we can obtain a more comprehensive understanding of the intramolecular energy transfer dynamics of the PE dendrimers. For instance, we can directly establish the connections between spectroscopic signals and the coupled electronic-nuclear motions. However, the simulation of the time-resolved spectra on the basis of the on-the-fly nonadiabatic dynamics itself represents a challenging task. That is why simulations of such spectra with classical trajectories are rather limited, notably in comparison with a large number of simulations of population dynamics of molecular electronic states. Below we summarize the key results. Several groups performed ab initio classical trajectory simulations of time-resolved photoelectron spectra ${ }^{29-41}$ and time and frequency resolved fluorescence spectra. ${ }^{42-}$ 47 Computer simulations of transient absorption (TA) PP spectra were performed on several polyatomic chromophores by using different levels of ab initio theory and applying different theoretical methodologies. ${ }^{48-57}$ Recently, Gelin, Domcke and coworkers proposed a practical approach for the on-the-fly trajectory simulation of TA PP signals which is based on the quasiclassical approximation of the DW representation of nonlinear spectroscopy. ${ }^{58}$ The DW representation has been introduced into femtosecond spectroscopy by Yan, Fried, and Mukamel. ${ }^{59-}$ ${ }^{61}$ For well-separated pump and probe pulses (non-overlapping pulses), the DW representation provides a transparent understanding of the TA PP signals. The interaction between the pump/probe pulse and the system is described by the doorway/window operator. Between the pump and probe pulses, the dynamics evolution is fully governed by the field-free system Hamiltonian. The TA PP signal is obtained by averaging the product of the doorway operator (at the time moment zero) and 
the window operator (at the time delay between the pulses) over nuclear trajectories generated by the surface hopping or other protocol for the on-the-fly simulation of the nonadiabatic dynamics. This DW representation does not add too much additional effort to the on-the-fly nonadiabatic simulations. Furthermore, this approach provides a clear and direct correlation between the coupled electronic-nuclear motions in the energy transfer process and the time-resolved spectroscopic response.

In this work, we extended the DW representation of Ref. ${ }^{58}$ to account for polarizationsensitive detection of TA PP signals and combined it with the surface-hopping machinery for the on-the-fly simulation of TA PP spectra of PE dendrimers. A typical model system composed of meta-linked 2-ring and 3-ring units, as shown in Scheme 1, was taken as an example. The on-thefly nonadiabatic dynamics with Tully's fewest switch surface hopping (FSSH) ${ }^{62-69}$ was performed at the TDDFT level on the PE dendrimer model. The system was put in different initial electronic states, including the ground and low-lying excited states. Then, a series of dynamics calculations were performed with different initial states. During the dynamics simulations, the electronic-state energies and transition dipole moments (TDMs) between different electronic states were recorded. After the dynamics, the DW approach was used to simulate the TA PP spectra. Particularly, we examined the impact of polarizations of pump and probe pulses on TA PP signals.

All the dynamics calculations were performed with a developing version of the JADE-NAMD package. ${ }^{70-71}$ The Gaussian 16 package ${ }^{72}$ was used in the optimization and frequency analysis. The JADE-NAMD package interfaced with the Qchem 5.1 package $^{73}$ was developed to perform the 
TDDFT calculations in the on-the-fly nonadiabatic dynamics. Here the analytical nonadiabatic coupling vectors at the TDDFT level ${ }^{74-75}$ were obtained directly instead of the numerical calculations of the time-derivatives of the wavefunction overlaps. All components of the TA PP signals, including the ground-state bleach (GSB), stimulated emission (SE) and excited-state absorption (ESA), were calculated within the quasi-classical approximation of the DW representation for femtosecond spectroscopy. More detailed discussions on the Hamiltonian, nonlinear response theories, DW representation, quasiclassical approximation, on-the-fly surfacehopping simulation are given in the Supporting Information (SI).

This work provides the deep insight into microscopic mechanisms of photophysical processes responsible for the interconnection of the TA PP signals and the intramolecular excited-state energy transfer dynamics from 2-ring to 3-ring units of the PE dendrimer model. We believe that the current simulation tool based on on-the-fly nonadiabatic dynamics simulation and the DW representation of the time-resolved spectroscopy could also be employed to study more general excitation transfer processes widely existing in molecular systems or aggregates with multiple TDMs.

The geometrical structure of $\mathrm{S}_{0}$-min is shown in Scheme 1. The PE dendrimer model studied in this work is composed of 2-ring and 3-ring PE units. For each unit, the benzene rings are linked with ethynylene bonds, whose distances are labeled as $r_{1}, r_{2}$ and $r_{3}$ in sequence from left to right in Scheme 1. The molecule is planar at $\mathrm{S}_{0}$-min. To further discuss the polarized TA PP signals in this paper, the coordinate axes were set up. The $\mathrm{X}$-axis is parallel to the 3-ring unit, the $\mathrm{Y}$-axis is 
perpendicular to the $\mathrm{X}$-axis and lies within the molecular plane, and the $\mathrm{Z}$-axis is placed perpendicular to the molecular plane.

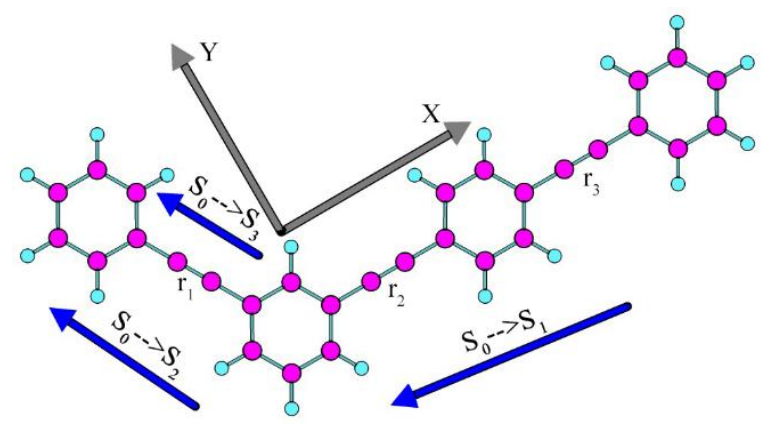

Scheme 1. The geometrical structure of the PE dendrimer model at $\mathrm{S}_{0}$-min. The blue arrows indicate the directions of the TDMs from $\mathrm{S}_{0}$ to the three low-lying excited states. The gray arrows indicate the directions of the coordinate axes, with the Z-axis perpendicular to the molecular plane.

The excited states at $\mathrm{S}_{0}$-min were calculated at the TDDFT/CAM-B3LYP level with 6-31G basis set, as shown in Table 1. The frontier molecular orbitals at $\mathrm{S}_{0}$-min are shown in Figure $\mathrm{S} 1$, SI, and their contributions to the excited state characters are shown in Table 1. $\mathrm{S}_{1}$ is mainly composed of the local excitation at the 3-ring unit, while $S_{2}$ is composed of significant contributions of the local excitation configuration at the 2-ring unit and a weaker component of the charge-transfer (CT) excitation between 2-ring and 3-ring units. $\mathrm{S}_{3}$ is mainly assigned as a CT state, and the residual TDM here may be due to the existence of the orbital overlap at the middle benzene ring. Although the electronic transition of $\mathrm{S}_{4}$ is localized at the 3-ring unit, the TMD is nearly zero due to orbital symmetry. 
Table 1. The vertical excited energies (VEEs), TDMs and electronic characters of the four lowlying electronic states at the TDDFT/CAM-B3LYP/6-31G level at $\mathrm{S}_{0}$-min of PE dendrimer model. For electronic configurations, only the components with contributions more than $10 \%$ are included.

\begin{tabular}{|c|c|c|c|c|c|c|c|}
\hline & \multirow[t]{2}{*}{$\operatorname{VEE}(\mathrm{eV})$} & \multicolumn{4}{|c|}{ TDM (Debye) } & \multicolumn{2}{|c|}{ Dominant Contributions } \\
\hline & & $\mathrm{X}$ & $\mathrm{Y}$ & $\mathrm{Z}$ & Tot & & \\
\hline$S_{1}$ & 4.03 & 12.09 & -1.36 & 0 & 12.17 & HOMO $\rightarrow$ LUMO & $82.5 \%$ \\
\hline \multirow[t]{2}{*}{$\mathrm{S}_{2}$} & 4.64 & 2.88 & -5.97 & 0 & 6.63 & HOMO-1 $\rightarrow$ LUMO+1 & $58.9 \%$ \\
\hline & & & & & & HOMO-1 $\rightarrow$ LUMO & $21.1 \%$ \\
\hline \multirow[t]{3}{*}{$\mathrm{S}_{3}$} & 4.71 & 0.77 & -1.41 & 0 & 1.61 & $\mathrm{HOMO} \rightarrow \mathrm{LUMO}+1$ & $28.4 \%$ \\
\hline & & & & & & HOMO-1 $\rightarrow$ LUMO & $17.5 \%$ \\
\hline & & & & & & $\mathrm{HOMO}-1 \rightarrow \mathrm{LUMO}+2$ & $11.4 \%$ \\
\hline \multirow[t]{2}{*}{$\mathrm{S}_{4}$} & 4.99 & 0 & -0.09 & 0 & 0.09 & $\mathrm{HOMO} \rightarrow \mathrm{LUMO}+3$ & $43.0 \%$ \\
\hline & & & & & & HOMO-5 $\rightarrow$ LUMO & $41.5 \%$ \\
\hline
\end{tabular}

From the electronic-structure point of view, the photoexcitation may bring the system to $\mathrm{S}_{2}$, which contains a significant local excitation component at the 2-ring unit. At the same time, $\mathrm{S}_{3}$ may be weakly populated by the excitation at a similar wavelength due to the weak oscillator strength and the vibronic-borrowing electronic transition. Then if the nonadiabatic dynamics takes place and the system quickly goes back to $\mathrm{S}_{1}$ dominated by the local excitation component at the 3-ring unit, the ultrafast excited-state energy transfer occurs from the short-chain to long-chain units.

The directions of TDMs from the ground to the first three excited states are shown in Scheme 1. The direction of TDM of $S_{1}$ is nearly aligned with two CC triple bonds of the 3-ring unit, while the directions of TDMs of $S_{2}$ and $S_{3}$ are nearly along with the CC triple bond of the 2-ring unit. This is consistent with the fact that $S_{1}$ and $S_{2}$ are mainly composed of the LE states located at the 
3-ring and 2-ring units, respectively. No TDM component was observed along the Z-axis that is perpendicular to the molecular plane.

To analyze the TA PP signals more conveniently, we plotted the TDMs between different electronic states at $\mathrm{S}_{0}$-min, see Figure 1. The TDM components in the $\mathrm{X}$-axis and $\mathrm{Y}$-axis directions are presented separately. The relation between the TDMs and signals is further discussed in the rest of this paper.
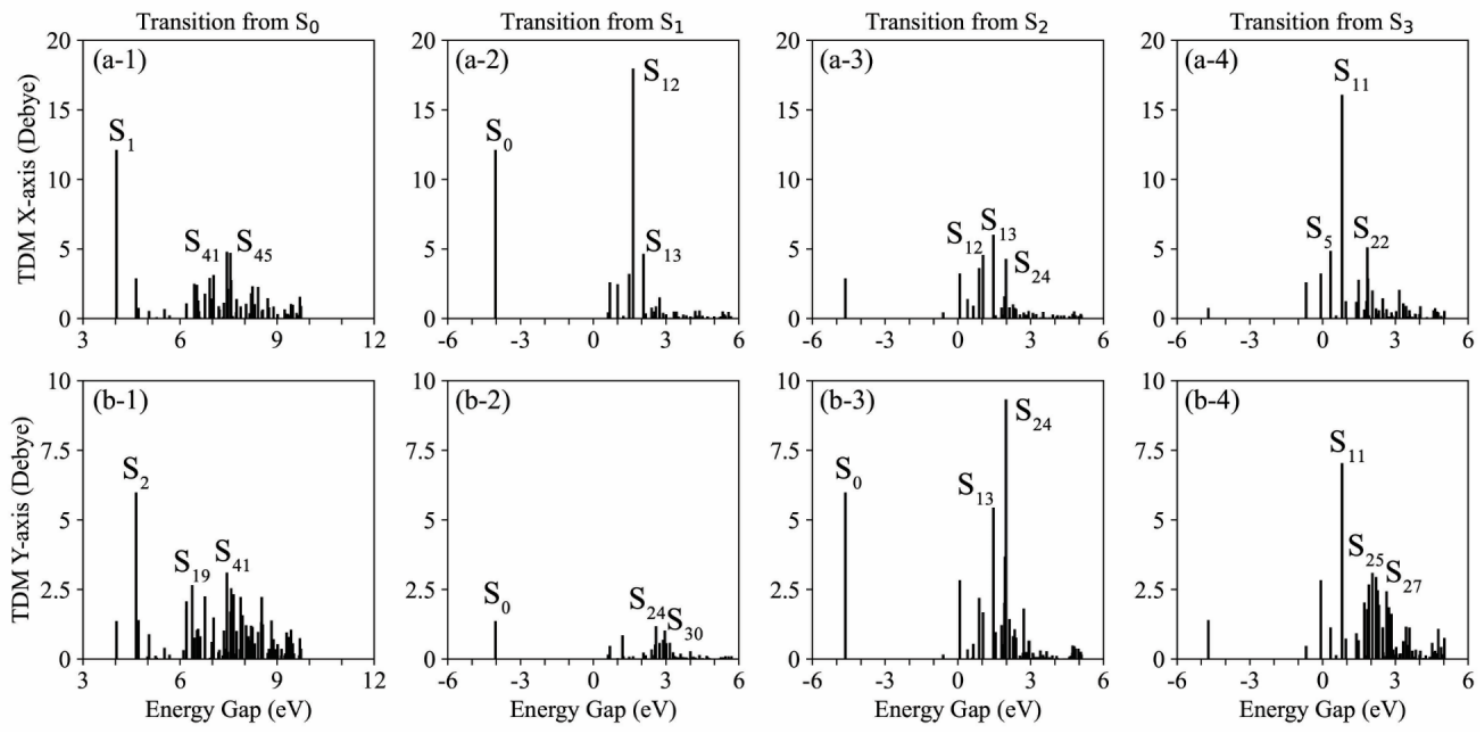

Figure 1. Absolute values of transition dipole moments (TDMs) in the (a) X-axis and (b) Y-axis direction from (1) $S_{0}$, (2) $S_{1}$, (3) $S_{2}$ and (4) $S_{3}$ to other electronic states at $S_{0}$-min. The three largest TDMs are assigned. 150 states are involved in the TDDFT calculations at the CAM-B3LYP/6$31 \mathrm{G}$ level.

To performe on-the-fly simulations of the TA PP signals and to facilitate interpretations of the obtained results, we have grouped the molecular states into three energetically well-separated 
manifolds $\{0\},\{I\}$ and $\{$ II $\}$, where $\{0\}$ is the electronic ground state; $\{$ I $\}$ comprises the states which can be interrogated by the pump pulse from the electronic ground state as well as other states which are dynamically (nonadiabatically) coupled to these states; $\{$ II $\}$ includes the states which can be interrogated by the probe pulse from the manifold $\{\mathrm{I}\}$. In this work, the lowest four excited states $\left(S_{1}, S_{2}, S_{3}\right.$ and $\left.S_{4}\right)$ were included in manifold $\{I\}$, and other 146 excited states with VEEs up to $10 \mathrm{eV}$ were incorporated in manifold $\{\mathrm{II}\}$.

The on-the-fly nonadiabatic dynamics simulations for the PE dendrimer model were performed assuming initial excitation in the states $S_{1}, S_{2}$, and $S_{3}$. Excitation to $S_{4}$ was not considered because this state has a vanishing TDM. Nonadiabatic transitions among all 4 states of manifold $\{\mathrm{I}\}$ were allowed for completeness. The time-dependent electronic populations are shown in Figure 2.
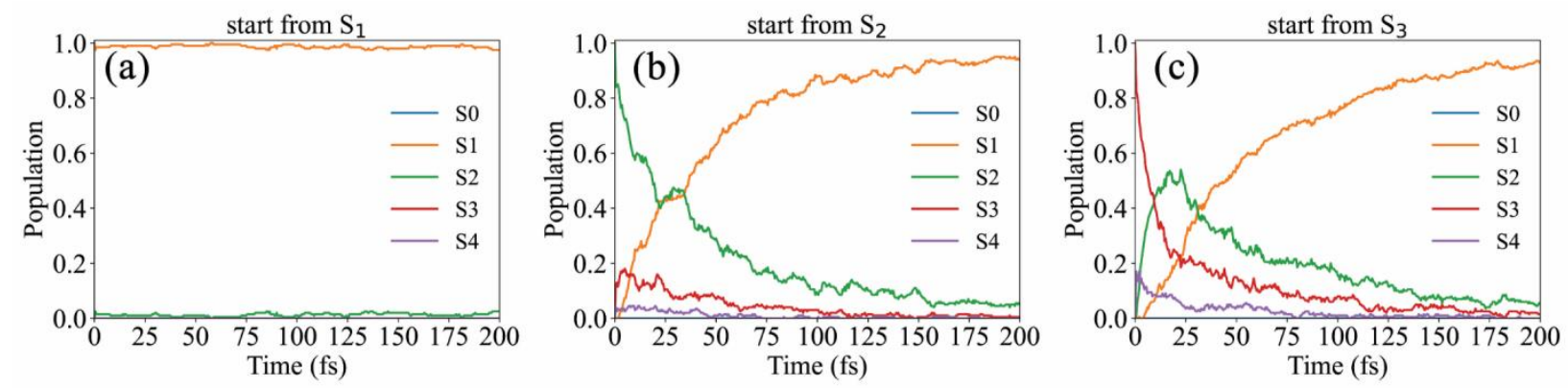

Figure 2. The time-dependent electronic populations of $\mathrm{S}_{0}-\mathrm{S}_{4}$ in the on-the-fly surface hopping nonadiabatic dynamics, which start from (a) $S_{1}$, (b) $S_{2}$ and (c) $S_{3}$, respectively. 200 trajectories were used for each initial state.

When the initial state is $S_{1}$, see Figure 2(a), most trajectories stay at this state during the whole 
dynamics. When the initial state is $S_{2}$, see Figure 2(b), the system decays to $S_{1}$ rapidly. About a half of the trajectories decay to $S_{1}$ within $35 \mathrm{fs}$, and most trajectories decay to $S_{1}$ at the end of simulation time $200 \mathrm{fs}$. The higher excited states, $\mathrm{S}_{3}$ and $\mathrm{S}_{4}$, are weakly populated in the early stage of dynamics. When the initial state is $S_{3}$, see Figure 2(c), the system first decays to $S_{2}$ very rapidly, and then $S_{1}$ is populated. Nearly half of the trajectories reach $S_{1}$ at 45 fs. Similar to the case when dynamics starts from $S_{2}$, most of the trajectories decay to $S_{1}$ at the end of simulation time.

The time-dependent electronic populations show that the system quickly decays to $S_{1}$ if the trajectories start from $S_{2}$ and $S_{3}$, which agrees well with previous on-the-fly nonadiabatic simulations at the semiempirical level of theory. ${ }^{18,22,28}$ As presented before, $S_{1}$ is nearly a LE state at the 3-ring unit, $\mathrm{S}_{2}$ is a hybrid LE state at the 2-ring unit and CT state between the 2-ring and 3ring units. The fast nonadiabatic decay from the $S_{2}$ to $S_{1}$ leads to the ultrafast excited-state energy transfer from the short-conjugated unit to the long-conjugated unit. In addition, we also noticed that the $S_{1}$ and $S_{2}$ energy gap becomes very small at the long $r_{1}$ distance [Figure $\mathrm{S} 2(\mathrm{a}-1), \mathrm{SI}$ ], and thus the $r_{1}$ stretching motion [Figure S3(a-3), SI] drives the nonadiabatic transition from $S_{2}$ to $S_{1}$. This observation is also highly consistent with the previous works. ${ }^{18,22,28}$

The TA PP signals of the PE dendrimer model were simulated using the DW representation of nonlinear response as described in Ref. ${ }^{58}$ and detailed in sections S1-S5 of SI.

We assume that the PE dendrimers are aligned (e.g. by the incorporation into a matrix/film or by the application of an external field) in the reference frame of Scheme 1 and study 4 variants of the polarizations $\left(\varepsilon_{p u}, \varepsilon_{p r}\right)$ of the pump and probe pulses along the axes $\mathrm{X}$ and $\mathrm{Y}$ : (X-X), (X- 
$\mathrm{Y}),(\mathrm{Y}-\mathrm{Y})$, and (Y-X). Pump pulses with two different frequencies, $\omega_{p u}=4.03 \mathrm{eV}$ and $4.64 \mathrm{eV}$ resonant with $S_{1}$ and $S_{2}$, were considered in the simulation. Notice that the energies of $S_{2}(4.64 \mathrm{eV})$ and $\mathrm{S}_{3}(4.71 \mathrm{eV})$ are very close, so the $\mathrm{S}_{3}$ contribution was also taken into account in the simulation. In this section, we will give a detailed analysis of the integral TA PP signals. The dispersed signals contain the same information and are presented in the SI (Figure S4 and S5). In the simulation of TA PP signals, the pump and probe pulses with Gaussian envelopes were used, namely $E_{a}(t)=\exp \left\{-\left(t / \tau_{a}\right)^{2}\right\}$ and $E_{a}(\omega)=\exp \left\{-\left(\omega \tau_{a}\right)^{2} / 4\right\}$ and the pulse durations $\tau_{a}$ were set to 5 fs $(\mathrm{a}=p u, p r)$.

The integral signal excited with $\omega_{p u}=4.03 \mathrm{eV}$ is shown in Figure 3. From left to right, the panels correspond to the GSB, SE, ESA contributions and the total integral signal. From top to bottom, the panels correspond to the polarizations of the pump and probe pulses $\left(\boldsymbol{\varepsilon}_{p u}, \boldsymbol{\varepsilon}_{p r}\right)$ along the axes $(\mathrm{X}-\mathrm{X}),(\mathrm{X}-\mathrm{Y}),(\mathrm{Y}-\mathrm{Y})$, and $(\mathrm{Y}-\mathrm{X})$ of the reference frame shown in Scheme 1. 

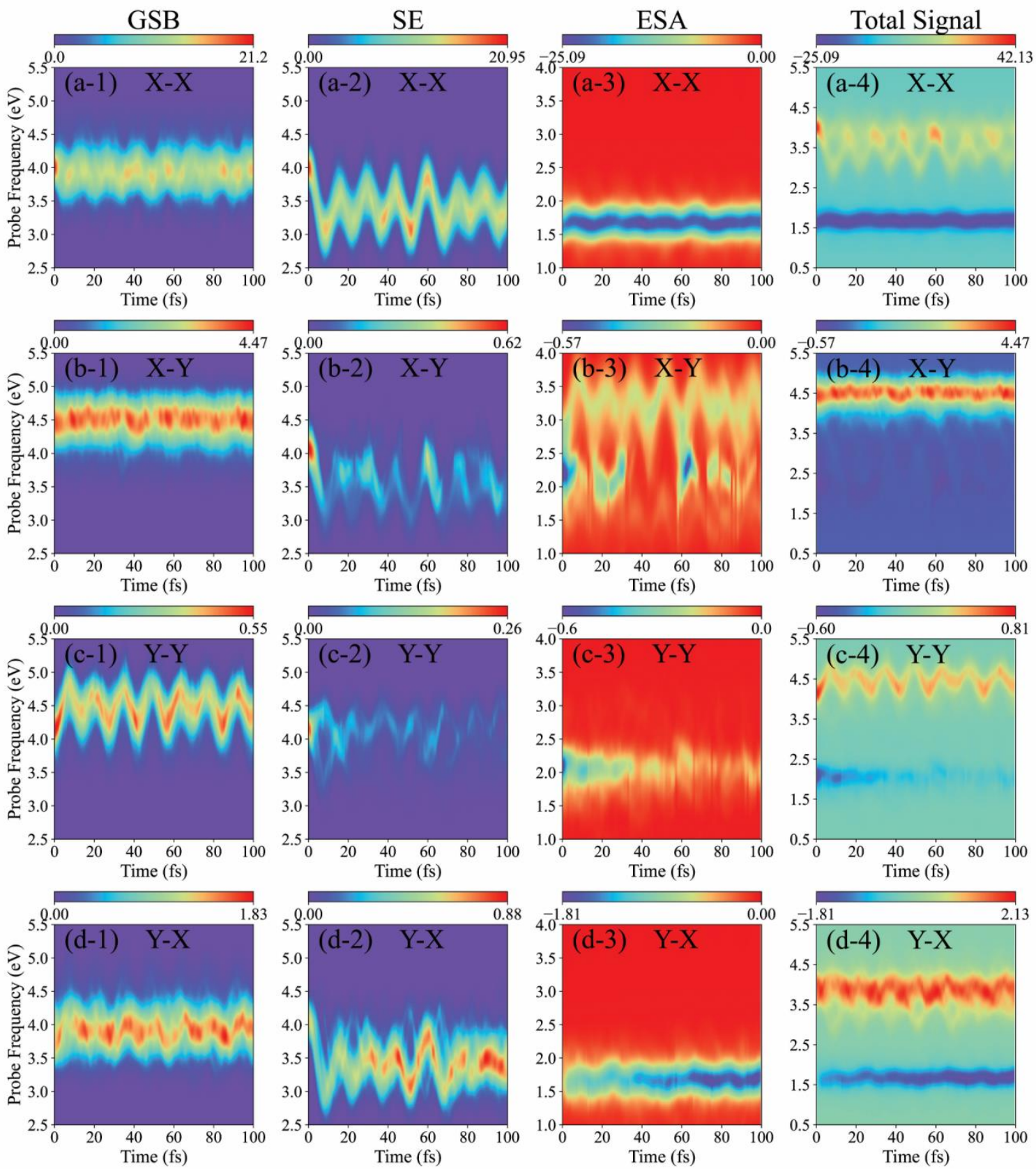

Figure 3. (1) GSB, (2) SE, (3) ESA contributions and (4) total integral signal of $I_{\mathrm{int}}\left(\tau, \omega_{p r}\right)$ as a function of $\tau$ and $\omega_{p r}$. The notation $\alpha-\beta(\alpha, \beta=\mathrm{X}, \mathrm{Y})$ in each panel indicates that the pump pulse polarization $\boldsymbol{\varepsilon}_{p u}$ is along the $\alpha$-axis direction, while the probe pulse polarization $\boldsymbol{\varepsilon}_{p r}$ is along the $\beta$-axis direction. The pump pulse with $\omega_{p u}=4.03 \mathrm{eV}$ is used. 
When the central frequency of the pump pulse is resonant with $S_{1}$, the excitation mainly brings the system into $S_{1}$. The TDM from $S_{0}$ to $S_{1}$ is mainly along the $X$-axis: $\mu_{0,1}^{x}=12.09$ Debye and $\mu_{0,1}^{y}=-1.36$ Debye (see Table 1). As a result, only the pump pulse with $\boldsymbol{\varepsilon}_{p u} \| \mathrm{X}$ excites the $\mathrm{S}_{1}$ state significantly.

Let us first consider the TA PP signal when the polarization of the probe pulse is also along the $\mathrm{X}$-axis direction $\left(\varepsilon_{p r} \| \mathrm{X}\right)$, as shown in Figure 3(a). In Figure 3(a-1), the GSB signal manifests electronic transitions between $\mathrm{S}_{0}$ and $\mathrm{S}_{1}$ and exhibits low-amplitude oscillations around $\omega_{p r} \sim 4.0$ $\mathrm{eV}$ and. Such oscillations reveal the high-frequency $\mathrm{CC}$ triplet bond stretching mode with a period $\sim 15$ fs and are caused by the combination of non-Condon effects, anharmonic effects, and finite pulse duration.

The SE signal, which is shown in Figure 3(a-2), gives important information on the excitedstate wavepacket motion. After being pumped, the system remains on the $S_{1}$ potential energy surface (PES) according to the population dynamics in Figure 2(a). In this case, the SE signal reflects the projection of the time-dependent excited-state wavepacket motion in the lowest excited electronic state to the electronic ground state. At $\tau=0$, the maximum of the SE signal corresponds to $\omega_{p r} \sim 4.0 \mathrm{eV}$. Then the "center of gravity" of the SE signal moves to the red and oscillates around $\sim 3.5 \mathrm{eV}$, which is close to the VEE of the $\mathrm{S}_{1}$ state at $\mathrm{S}_{1}$-min, see Table $\mathrm{S} 2$ in SI. Similar to the GSB signals, the oscillation of the SE signal is mainly due to the evolution of the $\mathrm{S}_{1}$ VEE under the stretching vibration of the CC triplet bonds $\left(\mathrm{r}_{2}\right.$ and $\left.\mathrm{r}_{3}\right)$ at the 3-ring unit. As discussed above, the significant elongations of $r_{2}$ and $r_{3}$ take place from $S_{0}$-min to $S_{1}$-min, see Table $S 2$ in 
SI. To clarify this argument, we examined the time-dependent evolution of the symmetric and asymmetric combinations of stretching vibrations of $r_{2}$ and $r_{3}$, and found that the symmetric stretching vibration [Figure S3(d-2), SI] gives the same oscillation patterns as the SE signal. This indicates that the symmetric stretching vibration of the $\mathrm{CC}$ triplet bonds at the 3-ring unit drives the system to move forward and backward with respect to the geometry of $S_{1}$-min, producing highamplitude SE oscillations. No SE decay was observed in the whole simulation time, which is consistent with the fact that the system always stays on $S_{1}$ if the dynamics starts from $S_{1}$, see Figure 2(a).

The ESA signal is shown in Figure 3(a-3). This signal reflects the projection of the wavepacket motion in the $S_{1}$ state to the high-lying excited states of manifold $\{$ II $\}$. The ESA signal is centered at $\omega_{p r} \sim 1.7 \mathrm{eV}$, which reveals the $\mathrm{S}_{1}$ to $\mathrm{S}_{12}$ transition having a much larger TDM component in the $\mathrm{X}$-axis direction than other $\{\mathrm{I}\} \rightarrow\{\mathrm{II}\}$ transitions, see Figure 1(a-2). A low-amplitude ESA signal oscillation with a period of 15 fs reveals the same CC triplet bond stretching.

The total signal, which is the sum of the GSB, SE and ESA contributions, is shown in Figure 3(a-4). Although the GSB and SE signals show a little overlap, they can be easily distinguished from the ESA signal because the latter one is located in a different spectral domain. In this sense, this system is a promising candidate for the future experimental TA PP study because the ESA signal does not overlap with the GSB and SE signals.

Next, we will discuss the integral TA PP signal for $\boldsymbol{\varepsilon}_{p u} \| \mathrm{X}$ and $\boldsymbol{\varepsilon}_{p r} \| \mathrm{Y}$, which is shown in 
Figure 3(b). As discussed above, the Y-component of the TDM from $\mathrm{S}_{0}$ to $\mathrm{S}_{2},\left(\mu_{0,2}^{y}=-5.97\right.$ Debye $)$ is almost four times larger than the $\mathrm{Y}$-component of the TDM from $\mathrm{S}_{0}$ to $\mathrm{S}_{1},\left(\mu_{0,1}^{y}=-1.36\right.$ Debye $)$. Hence the GSB signal [Figure 3(b-1)] is centered at $\omega_{p r} \sim 4.5 \mathrm{eV}$, close to the VEE of $\mathrm{S}_{2}$ in the Franck-Condon (FC) region, see Table S2 in SI. Therefore, the GSB signal is dominated by the electronic transition to $\mathrm{S}_{2}$. Since $\mu_{0,1}^{y}<\mu_{0,1}^{x}$, the $\mathrm{SE}$ signal shown in Figure 3(b-2) is much weaker than that in Figure 3(a-2). Similar to the TA PP signals with $\varepsilon_{p r} \| X$, the oscillation of the present SE signal reflects the wavepacket motion in the $\mathrm{S}_{1}$ state.

The ESA signal in Figure 3(b-3) is due to the transitions from $\mathrm{S}_{1}$ to several higher excited states, which are induced by the probe pulse with $\boldsymbol{\varepsilon}_{p r} \| \mathrm{Y}$. This signal differs considerably from its counterpart induced by the probe pulse with $\boldsymbol{\varepsilon}_{p r} \| \mathrm{X}$ shown in Figure 3(a-3). The reason is that the Y-components of the TDMs from $S_{1}$ to several higher excited states $\left(S_{4}, S_{24}, S_{30}\right)$ are rather similar, as shown in Figure 1(b-2). As a result, the ESA signal becomes diffuse in the probe frequency domain from $1.7 \mathrm{eV}$ to $3.7 \mathrm{eV}$.

The total signal for the Y-polarized probe pulse is shown in Figure 3(b-4), in which the GSB contribution dominates. At the same time, the GSB signal is well separated from the SE and ESA signals, while the late two components overlap with each other, results in the partial cancelation of the signal at 2.5 to $4.0 \mathrm{eV}$.

When the polarization of the pump pulse is along the $\mathrm{Y}$-axis direction, namely $\boldsymbol{\varepsilon}_{p u} \| \mathrm{Y}$, as shown in Figure 3(c) and (d), the signals become more complicated since $\mathrm{S}_{2}$ is also involved in the Y-axis excitation. However, it is not worthwhile to discuss them in detail, because the intensities 
of these signals are much weaker than those with $\boldsymbol{\varepsilon}_{p u} \| \mathrm{X}$.

It is necessary to point out that the intensity of the GSB, SE and ESA contributions for $\boldsymbol{\varepsilon}_{p u}=\boldsymbol{\varepsilon}_{p r} \| \mathrm{X}$ [Figure 3(a)] is much stronger than that for other combinations of the polarization vectors [Figure 3(b), (c) and (d)] because of much weaker TDM components. Thus, the total TA PP signal is also dominated by the X-X contribution depicted in Figure 3(a-4).

The integral signal for $\omega_{p u}=4.64 \mathrm{eV}$ is shown in Figure 4. In this case, the pump frequency is resonant with the $S_{0} \rightarrow S_{2}$ transition. Here the $S_{0} \rightarrow S_{2}$ TDM has X-and Y-components of comparable intensity: $\mu_{0,2}^{x}=2.88$ Debye and $\mu_{0,2}^{y}=-5.97$ Debye (see Table 1 ). Thus TA PP signals have considerable intensity for any polarization of pump and probe pulses [Figure 4(a), (b), (c) and (d)]. That is why the signals with $\boldsymbol{\varepsilon}_{p u} \| \mathrm{X}$ and $\boldsymbol{\varepsilon}_{p u} \| \mathrm{Y}$ are qualitatively similar. For the sake of simplicity, we focus on the analysis of the signals with $\boldsymbol{\varepsilon}_{p u} \| \mathrm{Y}$ [Figure 4 (c) and (d)] because of $\mu_{0,2}^{y}>\mu_{0,2}^{x}$. 

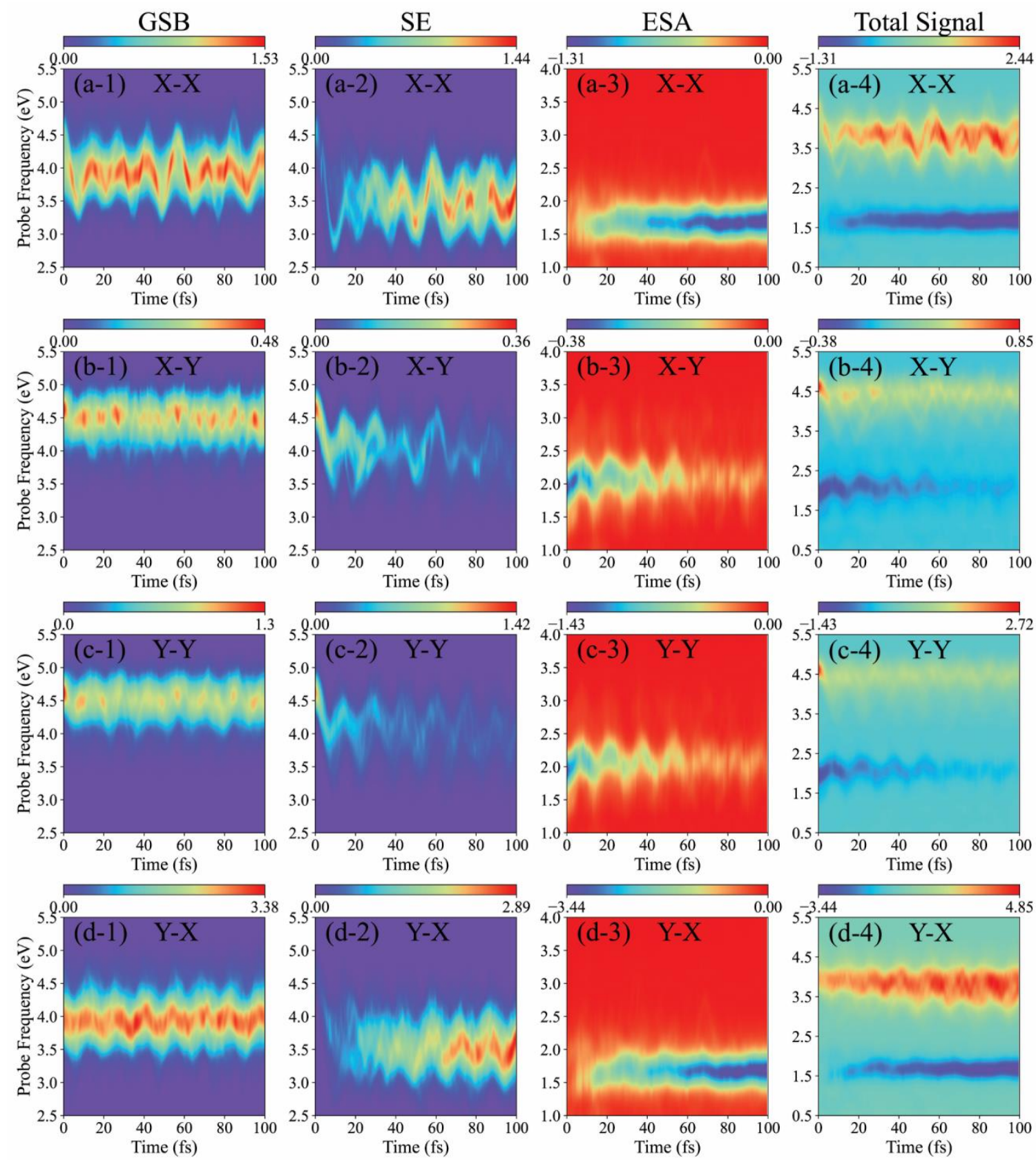

Figure 4. (1) GSB, (2) SE, (3) ESA contributions and (4) total integral signal of $I_{\mathrm{int}}\left(\tau, \omega_{p r}\right)$ as a function of $\tau$ and $\omega_{p r}$. The notation $\alpha-\beta(\alpha, \beta=\mathrm{X}, \mathrm{Y})$ in each panel indicates that the pump pulse polarization $\boldsymbol{\varepsilon}_{p u}$ is along the $\alpha$-axis direction, while the probe pulse polarization $\boldsymbol{\varepsilon}_{p r}$ is along the $\beta$-axis direction. The pump pulse with $\omega_{p u}=4.64 \mathrm{eV}$ is used. 
According to Eqs. 18 and 19 in SI, the GSB signal is always dominated by the strongest electronic transition caused by the probe pulse. The GSB signals in Figures 4(c-1) and 4(d-1) are dominated by the transitions to the states $\mathrm{S}_{2}$ and $\mathrm{S}_{1}$, for $\boldsymbol{\varepsilon}_{p r} \| \mathrm{Y}$ and $\boldsymbol{\varepsilon}_{p r} \| \mathrm{X}$, respectively. As a result, the GSB signals in Figures 4(c-1) and 4(d-1) are very similar to those in Figures 4(b-1) and 4(a-1). The minor difference is caused by slightly different doorway functions. In addition, the excitation of the $S_{2}$ state may also trigger the excitation of the $S_{3}$ state, due to the small energy gap between the two states and relatively high spectral width of $5 \mathrm{fs}$ pump and probe pulses. The SE signal in Figure 4(c-2) monitors the excited-state nonadiabatic dynamics initiated in the state $\mathrm{S}_{2}$. In the beginning, the SE maximum is located at $\omega_{p r} \sim 4.6 \mathrm{eV}$, which is close to the VEE of the $\mathrm{S}_{2}$ state in the FC region (Table S2, SI). Then the wavepacket starts moving on the $\mathrm{S}_{2} \mathrm{PES}$, and its motion is manifested through the oscillatory patterns of the SE signal. After a while, the wavepacket reaches the $S_{1} / S_{2}$ PES crossing and transfers to the $S_{1}$ state. Thus, the oscillatory SE signal becomes weaker with time and nearly disappears after 80 fs. The rapid quenching of the SE signal is consistent with the $\mathrm{S}_{2}$ population dynamics shown in Figure 2(b). Most importantly, while the SE signal for $\boldsymbol{\varepsilon}_{p r} \| \mathrm{Y}$ decays [Figure 4(c-2)], the SE signal for $\boldsymbol{\varepsilon}_{p r} \| \mathrm{X}$ rises [Figure 4(d-2)]. The reason is that the SE signal for $\boldsymbol{\varepsilon}_{p r} \|$ Y originates mainly from the local excitation at the 2ring unit, while the SE signal for $\boldsymbol{\varepsilon}_{p r} \| \mathrm{X}$ is dominated by the local excitation at the 3-ring unit. Therefore, quenching of the SE signal in the Y direction and simultaneous rise of the SE signal in the $\mathrm{X}$ direction is a clear fingerprint of the ultrafast energy transfer from the 2-ring to the 3-ring unit. 
The ESA signal for $\boldsymbol{\varepsilon}_{p r} \| \mathrm{Y}$ is shown in Figure 4(c-3). The signal reveals absorption from $\mathrm{S}_{2}$ to other high-lying states of manifold $\{$ II $\}$ at the 2-ring unit. The signal is located around $\omega_{p r} \sim$ $2.0 \mathrm{eV}$, which matches $\mathrm{S}_{2} \rightarrow \mathrm{S}_{24}$ transition caused by the TDM that has the strongest component in the Y direction, see Figure 1(b-3). Similar to the SE signal, the ESA signal exhibits a pronounced oscillatory pattern that reflects the nuclear motion on the $\mathrm{S}_{2}$ PES. In addition, the ESA intensity in the Y direction decreases with time [Figure 4(c-3)] while the ESA intensity in the X directions increases [Figure 4(d-3)]. This is another indication of the 2-ring to 3-ring energy transfer.

The total signals probed in the $\mathrm{Y}$ and $\mathrm{X}$ directions are shown in Figures $4(\mathrm{c}-4)$ and $4(\mathrm{~d}-4)$, respectively. The mechanism and timescale of the excited-state energy-transfer process can clearly be deduced by combining these two signals. The clearest picture is obtained by examining the ESA signal that is well separated from the GSB and SE signals: its vanishing in the Y direction and appearance in the $\mathrm{X}$ direction directly reflects the nonadiabatically driven excited-state energy transfer from the 2-ring unit to the 3-ring unit. In spite of the spectral overlap of the GSB and SE signals, we clearly monitor the same process because the GSB+SE signal in the $\mathrm{Y}(\mathrm{X})$ direction decreases (increases) with time. It should also be noticed that the SE signals in X and Y directions are located at different wavelengths. The SE signal decays in the higher-energy domain in the Y direction, while grows in the lower-energy domain in the $\mathrm{X}$ direction. This observation also reveals the excited-state energy transfer. This indicates that high time and frequency resolution in conjunction with polarization-sensitive detection of TA PP signals allows us to scrutinize excitedstate energy transfer in dendrimers. We thus suggest that, polarization-sensitive pump-probe 
experiments give a direct real-time fingerprint of intramolecular excited-state energy transfer if the involved localized electronic states possess different energies and different TDM orientations.

In this work, we simulated the TA PP signals of the PE dendrimer model system by combining the on-the-fly nonadiabatic dynamics and the DW representation of nonlinear spectroscopy. We incorporated polarizations of laser fields into the DW representation and simulated integral and dispersed TA PP signals for different polarizations of the pump and probe pulses

We carefully analyzed total TA PP signals as well as the GSB, SE and ESA contributions. When the pump pulse is resonant with the $S_{0} \rightarrow S_{1}$ transition and polarization of the pump pulse coincides with the $S_{0} \rightarrow S_{1}$ TDM direction (X-axis), $S_{1}$ is significantly populated and the excitedstate wavepacket dynamics driven by the stretching vibrations of the CC triple bonds is manifested in the high-amplitude oscillation of the SE signal. This oscillatory contribution is also pronounced when the probe pulse is polarized along the X-axis. The GSB signal depends significantly on the polarization of the probe pulse because different excited states have different TDM orientations. The ESA signal is well spectrally separated from the overlapping GSB/SE counterparts and, similar to the SE signal, exhibits vibrational oscillations revealing the CC triple bonds.

When the pump pulse is resonant with the $S_{0} \rightarrow S_{2}$ transition, $S_{2}$ is significantly populated for any polarization of the pump pulse. Next, a fast ( $40 \mathrm{fs}) \mathrm{S}_{2} \rightarrow \mathrm{S}_{1}$ internal conversion takes place, which corresponds to the excited-state energy transfer from the 2-ring to 3-ring units. The $\mathrm{S}_{0} \rightarrow \mathrm{S}_{1}$ TDM has a large component in the X-direction only, while the $\mathrm{S}_{0} \rightarrow \mathrm{S}_{2}$ TDM has comparable $\mathrm{X}$ and $\mathrm{Y}$ components. Therefore, the SE signal with the $\mathrm{Y}$-polarized probe quenches 
while the SE signal with the $\mathrm{X}$-axis polarized probe pulse rises. Similar information is encoded in the ESA signals. We thus conclude that the use of different pump-probe polarizations provides the direct spectroscopic evidence of the energy transfer from the short-conjugated unit to the longconjugated unit in dendrimers.

This work demonstrates that on-the-fly nonadiabatic dynamics simulation combined with the DW representation of nonlinear spectroscopy provides a promising approach to ab initio calculations of time-resolved TA PP spectra. In particular, this methodology gives a practical and numerically feasible tool to simulate time-resolved spectra of those polyatomic systems which exhibit nonadiabatic dynamics in lower-lying excited electronic states. In addition, this work clearly indicates that polarization-sensitive detection of TA PP signals in conjunction with ab initio on-the-fly simulation of these signals may provide a direct way to explore ultrafast energy-transfer in lower-lying optically-bright excited states possessing different TDM orientations. We hope that this idea may encourage additional experimental studies of similar systems in the future.

Supporting Information Available: Theoretical descriptions of the polarization-sensitive TA PP spectroscopy in the DW representation, quasi-classical evaluation, computational details, VEEs and TDMs along the stretching of CC triple bonds, time-dependent distributions of CC triple bonds, TA PP dispersed signals and long-time (200 fs) TA PP integral signals. 


\section{Author Information}

\section{Corresponding Author}

E-mail: zhenggang.lan@m.scnu.edu.cn; zhenggang.lan@gmail.com.

\section{Notes}

The authors declare no competing financial interest.

\section{Acknowledgments}

This work is supported by NSFC projects (No. 21933011 and 21873112). The authors thank the Supercomputing Center, Computer Network Information Center, Chinese Academy of Sciences; National Supercomputing Center in Shenzhen for providing computational resources. M.F.G. acknowledges the support of Hangzhou Dianzi University through the startup funding.

\section{References}

(1) Tretiak, S.; Mukamel, S. Density matrix analysis and simulation of electronic excitations in conjugated and aggregated molecules. Chem. Rev. 2002, 102, 3171-3212.

(2) Nelson, T.; Fernandez-Alberti, S.; Roitberg, A. E.; Tretiak, S. Nonadiabatic excited-state molecular dynamics: Modeling photophysics in organic conjugated materials. Acc. Chem. Res. 2014, 47, 1155-1164.

(3) Kilina, S.; Kilin, D.; Tretiak, S. Light-driven and phonon-assisted dynamics in organic and semiconductor nanostructures. Chem. Rev. 2015, 115, 5929-5978.

(4) Nelson, T. R.; White, A. J.; Bjorgaard, J. A.; Sifain, A. E.; Zhang, Y.; Nebgen, B.; Fernandez- 
Alberti, S.; Mozyrsky, D.; Roitberg, A. E.; Tretiak, S. Non-adiabatic excited-state molecular dynamics: Theory and applications for modeling photophysics in extended molecular materials. Chem. Rev. 2020, 120, 2215-2287.

(5) Xu, Z. F.; Kahr, M.; Walker, K. L.; Wilkins, C. L.; Moore, J. S. Phenylacetylene dendrimers by the divergent, convergent, and double-stage convergent methods. J. Am. Chem. Soc. 1994, 116, 4537-4550.

(6) Shortreed, M. R.; Swallen, S. F.; Shi, Z. Y.; Tan, W. H.; Xu, Z. F.; Devadoss, C.; Moore, J. S.; Kopelman, R. Directed energy transfer funnels in dendrimeric antenna supermolecules. J. Phys. Chem. B 1997, 101, 6318-6322.

(7) Swallen, S. F.; Zhu, Z. G.; Moore, J. S.; Kopelman, R. Correlated excimer formation and molecular rotational dynamics in phenylacetylene dendrimers. J. Phys. Chem. B 2000, 104, 3988 3995.

(8) Tada, T.; Nozaki, D.; Kondo, M.; Yoshizawa, K. Molecular orbital interactions in the nanostar dendrimer. J. Phys. Chem. B 2003, 107, 14204-14210.

(9) Shimoi, Y.; Friedman, B. A. A tight-binding model of phenylene molecules with metaconnections - implications for phenylacetylene dendrimers. Chem. Phys. 1999, 250, 13-22.

(10) Raychaudhuri, S.; Shapir, Y.; Chernyak, V. V.; Mukamel, S. Excitonic funneling in extended dendrimers with nonlinear and random potentials. Phys. Rev. Lett. 2000, 85, 282-285.

(11) Palma, J. L.; Atas, E.; Hardison, L.; Marder, T. B.; Collings, J. C.; Beeby, A.; Melinger, J. S.; Krause, J. L.; Kleiman, V. D.; Roitberg, A. E. Electronic spectra of the nanostar dendrimer: Theory and experiment. J. Phys. Chem. C 2010, 114, 20702-20712.

(12) Kleiman, V. D.; Melinger, J. S.; McMorrow, D. Ultrafast dynamics of electronic excitations in a light-harvesting phenylacetylene dendrimer. J. Phys. Chem. B 2001, 105, 5595-5598.

(13) Freixas, V. M.; Ondarse-Alvarez, D.; Tretiak, S.; Makhov, D. V.; Shalashilin, D. V.; Fernandez-Alberti, S. Photoinduced non-adiabatic energy transfer pathways in dendrimer building blocks. J. Chem. Phys. 2019, 150, 124301. 
(14) Fernandez-Alberti, S.; Roitberg, A. E.; Kleiman, V. D.; Nelson, T.; Tretiak, S. Shishiodoshi unidirectional energy transfer mechanism in phenylene ethynylene dendrimers. J. Chem. Phys. 2012, 137, 22A526.

(15) Atas, E.; Peng, Z.; Kleiman, V. D. Energy transfer in unsymmetrical phenylene ethynylene dendrimers. J. Phys. Chem. B 2005, 109, 13553-13560.

(16) Tortschanoff, A.; Mukamel, S. Pump-probe simulation study of the two-exciton manifold of dendrimers. J. Phys. Chem. A 2002, 106, 7521-7529.

(17) Kirkwood, J. C.; Scheurer, C.; Chernyak, V.; Mukamel, S. Simulations of energy funneling and time- and frequency-gated fluorescence in dendrimers. J. Chem. Phys. 2001, 114, 2419-2429. (18) Fernandez-Alberti, S.; Kleiman, V. D.; Tretiak, S.; Roitberg, A. E. Nonadiabatic molecular dynamics simulations of the energy transfer between building blocks in a phenylene ethynylene dendrimer. J. Phys. Chem. A 2009, 113, 7535-7542.

(19) Huang, J.; Du, L.; Hu, D.; Lan, Z. Theoretical analysis of excited states and energy transfer mechanism in conjugated dendrimers. J. Comput. Chem. 2015, 36, 151-163.

(20) Thompson, A. L.; Gaab, K. M.; Xu, J.; Bardeen, C. J.; Martínez, T. J. Variable electronic coupling in phenylacetylene dendrimers: The role of Förster, Dexter, and charge-transfer Interactions. J. Phys. Chem. A 2004, 108, 671-682.

(21) Gaab, K. M.; Thompson, A. L.; Xu, J. J.; Martinez, T. J.; Bardeen, C. J. Meta-conjugation and excited-state coupling in phenylacetylene dendrimers. J. Am. Chem. Soc. 2003, 125, 92889289.

(22) Fernandez-Alberti, S.; Makhov, D. V.; Tretiak, S.; Shalashilin, D. V. Non-adiabatic excited state molecular dynamics of phenylene ethynylene dendrimer using a multiconfigurational Ehrenfest approach. Phys. Chem. Chem. Phys. 2016, 18, 10028-10040.

(23) Ondarse-Alvarez, D.; Kömürlü, S.; Roitberg, A. E.; Pierdominici-Sottile, G.; Tretiak, S.; Fernandez-Alberti, S.; Kleiman, V. D. Ultrafast electronic energy relaxation in a conjugated dendrimer leading to inter-branch energy redistribution. Phys. Chem. Chem. Phys. 2016, 18, 
25080-25089.

(24) Alfonso Hernandez, L.; Nelson, T.; Gelin, M. F.; Lupton, J. M.; Tretiak, S.; Fernandez-Alberti, S. Interference of interchromophoric energy-transfer pathways in $\pi$-conjugated macrocycles. $J$. Phys. Chem. Lett. 2016, 7, 4936-4944.

(25) Swallen, S. F.; Kopelman, R.; Moore, J. S.; Devadoss, C. Dendrimer photoantenna supermolecules: energetic funnels, exciton hopping and correlated excimer formation. J. Mol. Struct. 1999, 485, 585-597.

(26) Galindo, J. F.; Atas, E.; Altan, A.; Kuroda, D. G.; Fernandez-Alberti, S.; Tretiak, S.; Roitberg, A. E.; Kleiman, V. D. Dynamics of energy transfer in a conjugated dendrimer driven by ultrafast localization of excitations. J. Am. Chem. Soc. 2015, 137, 11637-11644.

(27) Tretiak, S.; Chernyak, V.; Mukamel, S. Localized electronic excitations in phenylacetylene dendrimers. J. Phys. Chem. B 1998, 102, 3310-3315.

(28) Malone, W.; Nebgen, B.; White, A.; Zhang, Y.; Song, H.; Bjorgaard, J. A.; Sifain, A. E.; Rodriguez-Hernandez, B.; Freixas, V. M.; Fernandez-Alberti, S.; Roitberg, A. E.; Nelson, T. R.; Tretiak, S. NEXMD software package for nonadiabatic excited state molecular dynamics simulations. J. Chem. Theory Comput. 2020, 16, 5771-5783.

(29) Hudock, H. R.; Martínez, T. J. Excited-state dynamics of cytosine reveal multiple intrinsic subpicosecond pathways. Chemphyschem 2008, 9, 2486-2490.

(30) Werner, U.; Mitrić, R.; Suzuki, T.; Bonačić-Koutecký, V. Nonadiabatic dynamics within the time dependent density functional theory: Ultrafast photodynamics in pyrazine. Chem. Phys. 2008, 349, 319-324.

(31) Thompson, A. L.; Martinez, T. J. Time-resolved photoelectron spectroscopy from first principles: Excited state dynamics of benzene. Faraday Discuss. 2011, 150, 293-311.

(32) Humeniuk, A.; Wohlgemuth, M.; Suzuki, T.; Mitrić, R. Time-resolved photoelectron imaging spectra from non-adiabatic molecular dynamics simulations. J. Chem. Phys. 2013, 139, 134104.

(33) Ren, H.; Fingerhut, B. P.; Mukamel, S. Time resolved photoelectron spectroscopy of 
thioflavin T photoisomerization: A simulation study. J. Phys. Chem. A 2013, 117, 6096-6104. (34) Tomasello, G.; Humeniuk, A.; Mitric, R. Exploring ultrafast dynamics of pyrazine by timeresolved photoelectron imaging. J. Phys. Chem. A 2014, 118, 8437-8445.

(35) Arbelo-Gonzalez, W.; Crespo-Otero, R.; Barbatti, M. Steady and time-resolved photoelectron spectra based on nuclear ensembles. J. Chem. Theory Comput. 2016, 12, 5037-5049.

(36) Mignolet, B.; Kanno, M.; Shimakura, N.; Koseki, S.; Remacle, F.; Kono, H.; Fujimura, Y. Ultrafast nonradiative transition pathways in photo-excited pyrazine: Ab initio analysis of timeresolved vacuum ultraviolet photoelectron spectrum. Chem. Phys. 2018, 515, 704-709.

(37) Mai, S.; Mohamadzade, A.; Marquetand, P.; Gonzalez, L.; Ullrich, S. Simulated and experimental time-resolved photoelectron spectra of the intersystem crossing dynamics in 2thiouracil. Molecules 2018, 23, 2836.

(38) Piteša, T.; Sapunar, M.; Ponzi, A.; Gelin, M. F.; Došlić, N.; Domcke, W.; Decleva, P. Combined surface-hopping, Dyson orbital, and B-spline approach for the computation of timeresolved photoelectron spectroscopy signals: The internal conversion in pyrazine. J. Chem. Theory Comput. 2021.

(39) Glover, W. J.; Mori, T.; Schuurman, M. S.; Boguslavskiy, A. E.; Schalk, O.; Stolow, A.; Martinez, T. J. Excited state non-adiabatic dynamics of the smallest polyene, trans 1,3-butadiene. II. Ab initio multiple spawning simulations. J. Chem. Phys. 2018, 148, 164303.

(40) Ruckenbauer, M.; Mai, S.; Marquetand, P.; González, L. Revealing deactivation pathways hidden in time-resolved photoelectron spectra. Sci. Rep. 2016, 6, 35522.

(41) Chakraborty, P.; Liu, Y. S.; McClung, S.; Weinacht, T.; Matsika, S. Time resolved photoelectron spectroscopy as a test of electronic structure and nonadiabatic dynamics. J. Phys. Chem. Lett. 2021, 12, 5099-5104.

(42) Cusati, T.; Granucci, G.; Persico, M. Photodynamics and time-resolved fluorescence of azobenzene in solution: A mixed quantum-classical simulation. J. Am. Chem. Soc. 2011, 133, 5109-5123. 
(43) Klaumunzer, B.; Kroner, D.; Lischka, H.; Saalfrank, P. Non-adiabatic excited state dynamics of riboflavin after photoexcitation. Phys. Chem. Chem. Phys. 2012, 14, 8693-8702.

(44) Lan, Z.; Lu, Y.; Weingart, O.; Thiel, W. Nonadiabatic decay dynamics of a benzylidene malononitrile. J. Phys. Chem. A 2012, 116, 1510-1518.

(45) Pang, X. J.; Cui, X. Y.; Hu, D. P.; Jiang, C. W.; Zhao, D.; Lan, Z. G.; Li, F. L. "Watching" the dark state in ultrafast nonadiabatic photoisomerization process of a light-driven molecular rotary motor. J. Phys. Chem. A 2017, 121, 1240-1249.

(46) Roy, S.; Ardo, S.; Furche, F. 5-Methoxyquinoline Photobasicity Is Mediated by Water Oxidation. J. Phys. Chem. A 2019, 123, 6645-6651.

(47) Yu, J. K.; Liang, R. B.; Liu, F.; Martinez, T. J. First-Principles Characterization of the Elusive I Fluorescent State and the Structural Evolution of Retinal Protonated Schiff Base in Bacteriorhodopsin. J. Am. Chem. Soc. 2019, 141, 18193-18203.

(48) Polli, D.; Altoe, P.; Weingart, O.; Spillane, K. M.; Manzoni, C.; Brida, D.; Tomasello, G.; Orlandi, G.; Kukura, P.; Mathies, R. A.; Garavelli, M.; Cerullo, G. Conical intersection dynamics of the primary photoisomerization event in vision. Nature 2010, 467, 440-443.

(49) Nenov, A.; Borrego-Varillas, R.; Oriana, A.; Ganzer, L.; Segatta, F.; Conti, I.; Segarra-Marti, J.; Omachi, J.; Dapor, M.; Taioli, S.; Manzoni, C.; Mukamel, S.; Cerullo, G.; Garavelli, M. UVlight-induced vibrational coherences: The key to understand Kasha rule violation in transAzobenzene. J. Phys. Chem. Lett. 2018, 9, 1534-1541.

(50) Borrego-Varillas, R.; Teles-Ferreira, D. C.; Nenov, A.; Conti, I.; Ganzer, L.; Manzoni, C.; Garavelli, M.; Maria de Paula, A.; Cerullo, G. Observation of the sub-100 femtosecond population of a dark state in a thiobase mediating intersystem crossing. J. Am. Chem. Soc. 2018, 140, 1608716093.

(51) Picchiotti, A.; Nenov, A.; Giussani, A.; Prokhorenko, V. I.; Miller, R. J. D.; Mukamel, S.; Garavelli, M. Pyrene, a test case for deep-ultraviolet molecular photophysics. J. Phys. Chem. Lett. 2019, 10, 3481-3487. 
(52) Conti, I.; Cerullo, G.; Nenov, A.; Garavelli, M. Ultrafast spectroscopy of photoactive molecular systems from first principles: Where we stand today and where we are going. J. Am. Chem. Soc. 2020, 142, 16117-16139.

(53) Nguyen, T. S.; Koh, J. H.; Lefelhocz, S.; Parkhill, J. Black-box, real-time simulations of transient absorption spectroscopy. J. Phys. Chem. Lett. 2016, 7, 1590-1595.

(54) Bonafé, F. P.; Hernández, F. J.; Aradi, B.; Frauenheim, T.; Sánchez, C. G. Fully atomistic real-time simulations of transient absorption spectroscopy. J. Phys. Chem. Lett. 2018, 9, 43554359.

(55) Krumland, J.; Valencia, A. M.; Pittalis, S.; Rozzi, C. A.; Cocchi, C. Understanding real-time time-dependent density-functional theory simulations of ultrafast laser-induced dynamics in organic molecules. J. Chem. Phys. 2020, 153, 054106.

(56) Zimmermann, T.; Vaníček, J. Efficient on-the-fly ab initio semiclassical method for computing time-resolved nonadiabatic electronic spectra with surface hopping or Ehrenfest dynamics. J. Chem. Phys. 2014, 141, 134102.

(57) Begušić, T.; Roulet, J.; Vaníček, J. On-the-fly ab initio semiclassical evaluation of timeresolved electronic spectra. J. Chem. Phys. 2018, 149, 244115.

(58) Gelin, M. F.; Huang, X.; Xie, W.; Chen, L.; Doslic, N. A.; Domcke, W. Ab initio surfacehopping simulation of femtosecond transient-absorption pump-probe signals of nonadiabatic excited-state dynamics using the doorway-window representation. J. Chem. Theory Comput. 2021, 17, 2394-2408.

(59) Yan, Y. J.; Fried, L. E.; Mukamel, S. Ultrafast pump-probe spectroscopy: femtosecond dynamics in Liouville space. The Journal of Physical Chemistry 1989, 93, 8149-8162.

(60) Yan, Y. J.; Mukamel, S. Femtosecond pump-probe spectroscopy of polyatomic molecules in condensed phases. Phys. Rev. A 1990, 41, 6485-6504.

(61) Fried, L. E.; Mukamel, S. A classical theory of pump-probe photodissociation for arbitrary pulse durations. J. Chem. Phys. 1990, 93, 3063-3071. 
(62) Tully, J. C. Molecular dynamics with electronic transitions. J. Chem. Phys. 1990, 93, 10611071.

(63) Wang, L. J.; Akimov, A.; Prezhdo, O. V. Recent progress in surface hopping: 2011-2015. J. Phys. Chem. Lett. 2016, 7, 2100-2112.

(64) Crespo-Otero, R.; Barbatti, M. Recent advances and perspectives on nonadiabatic mixed quantum-classical dynamics. Chem. Rev. 2018, 118, 7026-7068.

(65) Mai, S.; Marquetand, P.; González, L. Nonadiabatic dynamics: The SHARC approach. WIREs Comput Mol Sci 2018, 8, e1370.

(66) Persico, M.; Granucci, G. An overview of nonadiabatic dynamics simulations methods, with focus on the direct approach versus the fitting of potential energy surfaces. Theor. Chem. Acc. 2014, 133, 1526.

(67) Curchod, B. F. E.; Martínez, T. J. Ab initio nonadiabatic quantum molecular dynamics. Chem. Rev. 2018, 118, 3305-3336.

(68) Barbatti, M.; Granucci, G.; Persico, M.; Ruckenbauer, M.; Vazdar, M.; Eckert-Maksić, M.; Lischka, H. The on-the-fly surface-hopping program system NEWTON-X: Application to ab initio simulation of the nonadiabatic photodynamics of benchmark systems. J Photoch Photobio A 2007, $190,228-240$.

(69) Aleotti, F.; Soprani, L.; Nenov, A.; Berardi, R.; Arcioni, A.; Zannoni, C.; Garavelli, M. Multidimensional potential energy surfaces resolved at the RASPT2 level for accurate photoinduced isomerization dynamics of azobenzene. J. Chem. Theory Comput. 2019, 15, 68136823.

(70) Hu, D.; Liu, Y. F.; Sobolewski, A. L.; Lan, Z. Nonadiabatic dynamics simulation of keto isocytosine: a comparison of dynamical performance of different electronic-structure methods. Phys. Chem. Chem. Phys. 2017, 19, 19168-19177.

(71) Du, L. K.; Lan, Z. G. An on-the-fly surface-hopping program JADE for nonadiabatic molecular dynamics of polyatomic systems: Implementation and applications. J. Chem. Theory 
Comput. 2015, 11, 1360-1374.

(72) Frisch, M. J.; Trucks, G. W.; Schlegel, H. B.; Scuseria, G. E.; Robb, M. A.; Cheeseman, J. R.; Scalmani, G.; Barone, V.; Petersson, G. A.; Nakatsuji, H.; Li, X.; Caricato, M.; Marenich, A. V.; Bloino, J.; Janesko, B. G.; Gomperts, R.; Mennucci, B.; Hratchian, H. P.; Ortiz, J. V.; Izmaylov, A. F.; Sonnenberg, J. L.; Williams; Ding, F.; Lipparini, F.; Egidi, F.; Goings, J.; Peng, B.; Petrone, A.; Henderson, T.; Ranasinghe, D.; Zakrzewski, V. G.; Gao, J.; Rega, N.; Zheng, G.; Liang, W.; Hada, M.; Ehara, M.; Toyota, K.; Fukuda, R.; Hasegawa, J.; Ishida, M.; Nakajima, T.; Honda, Y.; Kitao, O.; Nakai, H.; Vreven, T.; Throssell, K.; Montgomery Jr., J. A.; Peralta, J. E.; Ogliaro, F.; Bearpark, M. J.; Heyd, J. J.; Brothers, E. N.; Kudin, K. N.; Staroverov, V. N.; Keith, T. A.; Kobayashi, R.; Normand, J.; Raghavachari, K.; Rendell, A. P.; Burant, J. C.; Iyengar, S. S.; Tomasi, J.; Cossi, M.; Millam, J. M.; Klene, M.; Adamo, C.; Cammi, R.; Ochterski, J. W.; Martin, R. L.; Morokuma, K.; Farkas, O.; Foresman, J. B.; Fox, D. J. Gaussian 16 Rev. C.01, Wallingford, CT, 2016.

(73) Shao, Y.; Gan, Z.; Epifanovsky, E.; Gilbert, A. T. B.; Wormit, M.; Kussmann, J.; Lange, A. W.; Behn, A.; Deng, J.; Feng, X.; Ghosh, D.; Goldey, M.; Horn, P. R.; Jacobson, L. D.; Kaliman, I.; Khaliullin, R. Z.; Kus, T.; Landau, A.; Liu, J.; Proynov, E. I.; Rhee, Y. M.; Richard, R. M.; Rohrdanz, M. A.; Steele, R. P.; Sundstrom, E. J.; Woodcock, H. L., III; Zimmerman, P. M.; Zuev, D.; Albrecht, B.; Alguire, E.; Austin, B.; Beran, G. J. O.; Bernard, Y. A.; Berquist, E.; Brandhorst, K.; Bravaya, K. B.; Brown, S. T.; Casanova, D.; Chang, C.-M.; Chen, Y.; Chien, S. H.; Closser, K. D.; Crittenden, D. L.; Diedenhofen, M.; DiStasio, R. A., Jr.; Do, H.; Dutoi, A. D.; Edgar, R. G.; Fatehi, S.; Fusti-Molnar, L.; Ghysels, A.; Golubeva-Zadorozhnaya, A.; Gomes, J.; Hanson-Heine, M. W. D.; Harbach, P. H. P.; Hauser, A. W.; Hohenstein, E. G.; Holden, Z. C.; Jagau, T.-C.; Ji, H.; Kaduk, B.; Khistyaev, K.; Kim, J.; Kim, J.; King, R. A.; Klunzinger, P.; Kosenkov, D.; Kowalczyk, T.; Krauter, C. M.; Lao, K. U.; Laurent, A. D.; Lawler, K. V.; Levchenko, S. V.; Lin, C. Y.; Liu, F.; Livshits, E.; Lochan, R. C.; Luenser, A.; Manohar, P.; Manzer, S. F.; Mao, S.-P.; Mardirossian, N.; Marenich, A. V.; Maurer, S. A.; Mayhall, N. J.; Neuscamman, E.; Oana, C. M.; 
Olivares-Amaya, R.; O'Neill, D. P.; Parkhill, J. A.; Perrine, T. M.; Peverati, R.; Prociuk, A.; Rehn, D. R.; Rosta, E.; Russ, N. J.; Sharada, S. M.; Sharma, S.; Small, D. W.; Sodt, A.; Stein, T.; Stueck, D.; Su, Y.-C.; Thom, A. J. W.; Tsuchimochi, T.; Vanovschi, V.; Vogt, L.; Vydrov, O.; Wang, T.; Watson, M. A.; Wenzel, J.; White, A.; Williams, C. F.; Yang, J.; Yeganeh, S.; Yost, S. R.; You, Z.-Q.; Zhang, I. Y.; Zhang, X.; Zhao, Y.; Brooks, B. R.; Chan, G. K. L.; Chipman, D. M.; Cramer, C. J.; Goddard, W. A., III; Gordon, M. S.; Hehre, W. J.; Klamt, A.; Schaefer, H. F., III; Schmidt, M. W.; Sherrill, C. D.; Truhlar, D. G.; Warshel, A.; Xu, X.; Aspuru-Guzik, A.; Baer, R.; Bell, A. T.; Besley, N. A.; Chai, J.-D.; Dreuw, A.; Dunietz, B. D.; Furlani, T. R.; Gwaltney, S. R.; Hsu, C.P.; Jung, Y.; Kong, J.; Lambrecht, D. S.; Liang, W.; Ochsenfeld, C.; Rassolov, V. A.; Slipchenko, L. V.; Subotnik, J. E.; Van Voorhis, T.; Herbert, J. M.; Krylov, A. I.; Gill, P. M. W.; Head-Gordon, M. Advances in molecular quantum chemistry contained in the Q-Chem 4 program package. Mol. Phys. 2015, 113, 184-215.

(74) Zhang, X.; Herbert, J. M. Analytic derivative couplings for spin-flip configuration interaction singles and spin-flip time-dependent density functional theory. J. Chem. Phys. 2014, 141, 064104. (75) Ou, Q.; Fatehi, S.; Alguire, E.; Shao, Y.; Subotnik, J. E. Derivative couplings between TDDFT excited states obtained by direct differentiation in the Tamm-Dancoff approximation. $J$. Chem. Phys. 2014, 141, 024114 
Supporting information for

\section{The Spectral Fingerprint of Excited-State Energy Transfer in Dendrimers through Polarization-Sensitive Transient- Absorption Pump-Probe Signals: On-the-Fly Nonadiabatic Dynamics Simulations}

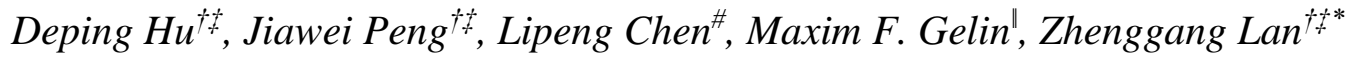

${ }^{\dagger}$ SCNU Environmental Research Institute, Guangdong Provincial Key Laboratory of Chemical Pollution and Environmental Safety \& MOE Key Laboratory of Environmental Theoretical Chemistry, South China Normal University, Guangzhou 510006, China

${ }^{+}$School of Environment, South China Normal University, Guangzhou 510006, China

\#Max Planck Institute for the Physics of Complex Systems, 01187 Dresden, Germany

${ }^{\|}$School of Sciences, Hangzhou Dianzi University, Hangzhou 310018, China

E-mail: zhenggang.lan@m.scnu.edu.cn; zhenggang.lan@gmail.com. 


\section{S1. Third-order nonlinear spectral}

Theoretical description of femtosecond spectroscopic signals starts, usually, from the total Hamiltonian $^{1}$

$$
\hat{H}(t)=\hat{H}_{M}+\hat{H}_{F}(t)
$$

where $\hat{H}_{M}$ is the Hamiltonian of the molecular system under study and

$$
\hat{H}_{F}(t)=-\hat{\boldsymbol{\mu}} \cdot \boldsymbol{E}(t)
$$

describes, in the dipole approximation, interaction of the system with the external fields ( $\hat{\boldsymbol{\mu}}$ is the TDM operator and $\boldsymbol{E}(t)$ is the total electric field of the laser pulses involved). The timedependent response of the molecular system on the external pulses is fully determined by the thirdorder polarization ${ }^{2}$

$$
\boldsymbol{P}^{(3)}(t)=(i)^{3} \int_{0}^{\infty} d t_{3} \int_{0}^{\infty} d t_{2} \int_{0}^{\infty} d t_{1} \boldsymbol{E}\left(t-t_{3}\right) \boldsymbol{E}\left(t-t_{3}-t_{2}\right) \boldsymbol{E}\left(t-t_{3}-t_{2}-t_{1}\right) S\left(t_{3}, t_{2}, t_{1}\right) .
$$

Here

$$
S\left(t_{3}, t_{2}, t_{1}\right)=\operatorname{Tr}\left\{\hat{\boldsymbol{\mu}}^{I}\left(t_{1}+t_{2}+t_{3}\right)\left[\hat{\boldsymbol{\mu}}^{I}\left(t_{1}+t_{2}\right),\left[\hat{\boldsymbol{\mu}}^{I}\left(t_{1}\right),\left[\hat{\boldsymbol{\mu}}^{I}(0), \hat{\rho}(-\infty)\right]\right]\right]\right\}
$$

is the total third-order response function, $\hat{\boldsymbol{\mu}}^{I}(t)=e^{i \hat{H}_{M}(t)} \hat{\boldsymbol{\mu}} e^{-i \hat{H}_{M}(t)}$ (hereafter, $\hbar=1$ ) and $\hat{\rho}(-\infty)$ is the system density operator before the arrival of the laser pulses at $t=-\infty$.

\section{S2. Transient-absorption pump-probe spectroscopy}

In TA PP spectroscopy, the molecular system is interrogated by pump ( $p u)$ and probe $(p r)$ pulses: 


$$
\begin{aligned}
& \boldsymbol{E}(t)=\boldsymbol{E}_{p u}(t)+\boldsymbol{E}_{p r}(t-\tau) \\
& \boldsymbol{E}_{p u}(t)=\boldsymbol{\varepsilon}_{p u} A_{p u} E_{p u}(t) e^{i k_{p u} x} e^{-i \omega_{p u} t}+c . c . \\
& \boldsymbol{E}_{p r}(t-\tau)=\boldsymbol{\varepsilon}_{p r} A_{p r} E_{p r}(t-\tau) e^{i \boldsymbol{k}_{p r} x} e^{-i \omega_{p r} t}+c . c .
\end{aligned}
$$

Here $\tau_{\tau}$ is the delay time between the pulses, while $\varepsilon_{a}, A_{a}, E_{a}(t), \boldsymbol{k}_{a}$ and $\omega_{a}$ are the unit vector of the polarization, amplitude, dimensionless envelope function, wave vector, and the carrier frequency of the pulse $(a=p u, p r)$.

The TA PP signal is determined by the third-order polarization in the phase-matching direction $\boldsymbol{k}_{p r}, P_{\boldsymbol{k}_{p r}}^{(3)}(\tau, t)$. Once this polarization is known, we can define the integral PP signal ${ }^{2}$

$$
I_{\text {int }}\left(\tau, \omega_{p r}\right)=\omega_{p r} \operatorname{Im}\left\{\int_{-\infty}^{\infty} d t E_{p r}(t) e^{i \omega_{p r} t} P_{k_{p r}}^{(3)}(\tau, t)\right\}
$$

and the dispersed PP signal ${ }^{2}$

$$
I_{d i s}(\tau, \omega)=\omega_{p r} \operatorname{Im}\left\{E_{p r}(\omega) P_{k_{p r}}^{(3)}(\tau, \omega)\right\}
$$

where $E_{p r}(\omega)$ and $P_{k_{p r}}^{(3)}(\tau, \omega)$ are the Fourier transforms of $E_{p r}(t)$ and $P_{k_{p r}}^{(3)}(\tau, t)$, respectively.

\section{S3. Molecular Hamiltonian}

To evaluate $I_{\mathrm{int}}\left(\tau, \omega_{p r}\right)$ and $I_{d i s}(\tau, \omega)$, we assume that the molecular Hamiltonian can be represented in the block-diagonal form:

$$
\hat{H}_{M}=\left(\begin{array}{ccc}
\hat{H}_{0} & 0 & 0 \\
0 & \hat{H}_{\mathrm{I}} & 0 \\
0 & 0 & \hat{H}_{\text {II }}
\end{array}\right)
$$

Here $\hat{H}_{0}$ is the nuclear Hamiltonian in the electronic ground state $\{0\}$; the Hamiltonian $\hat{H}_{\mathrm{I}}$ describes the manifold of the lower-lying electronic states $\{I\},{ }^{2-6}$ which are involved in the initial 
excitation and the nonadiabatic dynamics; the Hamiltonian $\hat{H}_{\mathrm{II}}$ describes vibronic dynamics in the manifold of the higher-lying states $\{$ II $\}$. In other words, we have grouped the molecular states into three energetically well-separated manifolds $\{0\},\{\mathrm{I}\}$ and $\{\mathrm{II}\}$, where $\{0\}$ is the electronic ground state; $\{\mathrm{I}\}$ comprises the states which can be interrogated by the pump pulse from the electronic ground state as well as other states which are dynamically (nonadiabatically) coupled to these states; $\{$ II $\}$ includes the states which can be interrogated by the probe pulse from the manifold $\{\mathrm{I}\}$. The TDM operators can therefore be written as a sum of the rising and lowering operators,

$$
\hat{\boldsymbol{\mu}}=\hat{\boldsymbol{\mu}}^{\uparrow}+\hat{\boldsymbol{\mu}}^{\downarrow}
$$

where

$$
\hat{\boldsymbol{\mu}}^{\uparrow}=\left(\begin{array}{ccc}
0 & \boldsymbol{\mu}_{0, \mathrm{I}} & 0 \\
0 & 0 & \boldsymbol{\mu}_{\mathrm{I}, \mathrm{II}} \\
0 & 0 & 0
\end{array}\right), \quad \hat{\boldsymbol{\mu}}^{\downarrow}=\left(\begin{array}{ccc}
0 & 0 & 0 \\
\boldsymbol{\mu}_{\mathrm{I}, 0} & 0 & 0 \\
0 & \boldsymbol{\mu}_{\mathrm{II}, \mathrm{I}} & 0
\end{array}\right)
$$

$\boldsymbol{\mu}_{0, \mathrm{I}}$ describes $\{0\} \rightarrow\{\mathrm{I}\}$ transitions and $\boldsymbol{\mu}_{\mathrm{I}, \mathrm{II}}$ describes $\{\mathrm{I}\} \rightarrow\{\mathrm{II}\}$ transitions. It is appropriate then to employ the rotating wave approximation (RWA) and rewrite the system-field interaction Hamiltonian in the form

$$
\hat{H}_{F}(t)=-\hat{\boldsymbol{\mu}}^{\uparrow} \boldsymbol{E}_{R W A}(t)-\hat{\boldsymbol{\mu}}^{\downarrow} \boldsymbol{E}_{R W A}^{*}(t)
$$

where

$$
\boldsymbol{E}_{R W A}(t)=\boldsymbol{\varepsilon}_{p u} A_{p u} E_{p u}(t) e^{-i \boldsymbol{k}_{p u} x} e^{i \omega_{p u} t}+\boldsymbol{\varepsilon}_{p r} A_{p r} E_{p r}(t-\tau) e^{-i \boldsymbol{k}_{p r} \boldsymbol{x}} e^{i \omega_{p r} t}
$$

The initial condition is given as

$$
\hat{\rho}(t=-\infty)=\hat{\rho}_{0,0}=|0\rangle\left\langle 0|\otimes| v_{0}\right\rangle\left\langle v_{0}\right|
$$


where $|0\rangle$ is the electronic ground state and $\left|v_{0}\right\rangle$ is the nuclear ground state (the lowest vibrational level).

\section{S4. Doorway-window approximation}

For obtaining operational expressions for the simulation of TA PP signals, we will make a series of approximations. Below we concentrate on the integral signal $I_{\text {int }}\left(\tau, \omega_{p r}\right)$. The dispersed signal can be treated very similarly, ${ }^{1}$ and we present the final formulas $I_{d i s}(\tau, \omega)$ at the end of this section. It is essential that the working expressions for the $\mathrm{D}$ and $\mathrm{W}$ functions listed below are generalized in comparison with those in Ref. ${ }^{1}$ to account for different polarizations of the pump and probe pulses.

First, we employed the DW approximation, ${ }^{2,4-6}$ which delivers an exact expression for $I_{\text {int }}\left(\tau, \omega_{p r}\right)$ if the pump and probe pulses are well separated, that is if $\tau$ is longer than the duration of the pump and probe pulses. Second, we adopted the short-pulse approximation, assuming that the laser pulses are short on the nuclear dynamics time scale. ${ }^{2}$ Within these assumptions, the integral PP signal can be cast in the form ${ }^{1}$

$$
I_{\mathrm{int}}\left(\tau, \omega_{p r}\right)=\omega_{p r} \operatorname{Tr}\left[\hat{D}_{0}\left(\omega_{p u}\right) e^{i \hat{H}_{0} \tau} \hat{W}_{0}\left(\omega_{p r}\right) e^{-i \hat{H}_{0} \tau}+\hat{D}_{\mathrm{I}}\left(\omega_{p u}\right) e^{i \hat{H}_{1} \tau}\left(\hat{W}_{\mathrm{I}}\left(\omega_{p r}\right)-\hat{W}_{\mathrm{II}}\left(\omega_{p r}\right)\right) e^{-i \hat{H}_{1} \tau}\right]
$$

where

$$
\begin{aligned}
& \hat{D}_{0}\left(\omega_{p u}\right)=\int_{-\infty}^{\infty} d t_{2}^{\prime} \int_{0}^{\infty} d t_{1} E_{p u}\left(t_{2}^{\prime}\right) E_{p u}\left(t_{2}^{\prime}-t_{1}\right) e^{i \omega_{p u} t_{1}} e^{-i \hat{H}_{\mathrm{I}} t_{1}} \boldsymbol{\varepsilon}_{p u} \boldsymbol{\mu}_{\mathrm{I}, 0} \hat{\rho}_{0,0} e^{i \hat{H}_{0} t_{1}} \boldsymbol{\varepsilon}_{p u} \boldsymbol{\mu}_{0, \mathrm{I}}+H . c . \\
& \hat{D}_{\mathrm{I}}\left(\omega_{p u}\right)=\int_{-\infty}^{\infty} d t_{2} \int_{0}^{\infty} d t_{1} E_{p u}\left(t_{2}^{\prime}\right) E_{p u}\left(t_{2}^{\prime}-t_{1}\right) e^{i \omega_{p u} t_{1}} \boldsymbol{\varepsilon}_{p u} \boldsymbol{\mu}_{0, \mathrm{I}} e^{-i \hat{H}_{1} t_{1}} \boldsymbol{\varepsilon}_{p u} \boldsymbol{\mu}_{\mathrm{I}, 0} \hat{\rho}_{0,0} e^{i \hat{H}_{0} t_{1}}+H . c .
\end{aligned}
$$

are the D-operators and 


$$
\begin{aligned}
& \hat{W}_{0}\left(\omega_{p r}\right)=\int_{-\infty}^{\infty} d t^{\prime} \int_{0}^{\infty} d t_{3} E_{p r}\left(t^{\prime}\right) E_{p r}\left(t^{\prime}+t_{3}\right) e^{i \omega_{p r} t_{3}} e^{i \hat{H}_{0} t_{3}} \boldsymbol{\varepsilon}_{p r} \boldsymbol{\mu}_{0, \mathrm{I}} e^{-i \hat{H}_{\mathrm{I}} t_{3}} \boldsymbol{\varepsilon}_{p r} \boldsymbol{\mu}_{\mathrm{I}, 0}+H . c . \\
& \hat{W}_{I}\left(\omega_{p r}\right)=\int_{-\infty}^{\infty} d t^{\prime} \int_{0}^{\infty} d t_{3} E_{p r}\left(t^{\prime}\right) E_{p r}\left(t^{\prime}+t_{3}\right) e^{i \omega_{p r} t_{3}} \boldsymbol{\varepsilon}_{p r} \boldsymbol{\mu}_{\mathrm{I}, 0} e^{i \hat{H}_{0} t_{3}} \boldsymbol{\varepsilon}_{p r} \boldsymbol{\mu}_{0, \mathrm{I}} e^{-i \hat{H}_{1} t_{3}}+H . c . \\
& \hat{W}_{\mathrm{II}}\left(\omega_{p r}\right)=\int_{-\infty}^{\infty} d t^{\prime} \int_{0}^{\infty} d t_{3} E_{p r}\left(t^{\prime}\right) E_{p r}\left(t^{\prime}+t_{3}\right) e^{i \omega_{p r} t_{3}} \boldsymbol{\varepsilon}_{p r} \boldsymbol{\mu}_{\mathrm{I}, \mathrm{I}} e^{-i \hat{H}_{\mathrm{II}} t_{3}} \boldsymbol{\varepsilon}_{p r} \boldsymbol{\mu}_{\mathrm{IIII}} e^{i \hat{H}_{\mathrm{I}} t_{3}}+H . c .
\end{aligned}
$$

are the W-operators. The terms proportional to $\hat{D}_{0} \hat{W}_{0}, \hat{D}_{\mathrm{I}} \hat{W}_{\mathrm{I}}, \hat{D}_{\mathrm{I}} \hat{W}_{\mathrm{II}}$ in Eq. (14) yield, correspondingly, the ground-state bleach (GSB), stimulated emission (SE), and excited-state absorption (ESA) contributions to the total TA PP signal.

\section{S5. Quasi-classical evaluation}

Eq. (14) delivers a fully quantum expression for $I_{\text {int }}\left(\tau, \omega_{p r}\right)$. In order to adopt it for the evaluation via surface hopping trajectory simulations, we resort to the following approximations. The DW operators become functions in the phase space of nuclear motion, the trace over nuclear degrees of freedom (DoFs) is replaced by the integral over the nuclear phase space, and quantum evolutions are approximated by evolutions along classical trajectories. We thus obtain:

$$
\begin{aligned}
& I_{\mathrm{int}}\left(\tau, \omega_{p r}\right)=I_{\mathrm{int}}^{G S B}\left(\tau, \omega_{p r}\right)+I_{\mathrm{int}}^{S E}\left(\tau, \omega_{p r}\right)+I_{\mathrm{int}}^{E S A}\left(\tau, \omega_{p r}\right) \\
& I_{\mathrm{int}}^{G S B}\left(\tau, \omega_{p r}\right)=\omega_{p r} \int d \boldsymbol{R}_{g} d \boldsymbol{P}_{g} D_{0}\left(\omega_{p u}, \boldsymbol{R}_{g}, \boldsymbol{P}_{g}\right) W_{0}^{\mathrm{int}}\left(\omega_{p r}, \boldsymbol{R}_{g}(\tau), \boldsymbol{P}_{g}(\tau)\right) \\
& I_{\mathrm{int}}^{S E}\left(\tau, \omega_{p r}\right)=\omega_{p r} \int d \boldsymbol{R}_{g} d \boldsymbol{P}_{g} D_{\mathrm{I}}\left(\omega_{p u}, \boldsymbol{R}_{g}, \boldsymbol{P}_{g}\right) W_{\mathrm{I}}^{\mathrm{int}}\left(\omega_{p r}, \boldsymbol{R}_{e}(\tau), \boldsymbol{P}_{e}(\tau)\right) \\
& I_{\mathrm{int}}^{E S A}\left(\tau, \omega_{p r}\right)=-\omega_{p r} \int d \boldsymbol{R}_{g} d \boldsymbol{P}_{g} D_{\mathrm{I}}\left(\omega_{p u}, \boldsymbol{R}_{g}, \boldsymbol{P}_{g}\right) W_{\mathrm{II}}^{\mathrm{int}}\left(\omega_{p r}, \boldsymbol{R}_{e}(\tau), \boldsymbol{P}_{e}(\tau)\right)
\end{aligned}
$$

Here $\boldsymbol{R}_{g}$ and $\boldsymbol{P}_{g}$ are the initial nuclear coordinates and momenta in the electronic ground state which are sampled from the Wigner distribution $\rho_{g}^{\text {Wig }}\left(\boldsymbol{R}_{g}, \boldsymbol{P}_{g}\right) . \boldsymbol{R}_{g}(\tau)$ and $\boldsymbol{P}_{g}(\tau)$ are the coordinates and momenta after propagation in the ground state up to $t=\tau$, while $\boldsymbol{R}_{e}(\tau)$ and $\boldsymbol{P}_{e}(\tau)$ are the coordinates and momenta after the trajectory propagation in the manifold of lower-lying 
excited states $\{\mathrm{I}\}$ up to $t=\tau$.

Finally

$$
\begin{aligned}
& D_{0}\left(\omega_{p u}, \boldsymbol{R}_{g}, \boldsymbol{P}_{g}\right)=\sum_{e}\left|\boldsymbol{\varepsilon}_{p u} \boldsymbol{\mu}_{g e}\left(\boldsymbol{R}_{g}\right)\right|^{2} E_{p u}^{2}\left[\omega_{p u}-U_{e g}\left(\boldsymbol{R}_{g}\right)\right] \rho_{g}^{W i g}\left(\boldsymbol{R}_{g}, \boldsymbol{P}_{g}\right) \\
& D_{\mathrm{I}}\left(\omega_{p u}, \boldsymbol{R}_{g}, \boldsymbol{P}_{g}\right)=\left|\boldsymbol{\varepsilon}_{p u} \boldsymbol{\mu}_{g e}\left(\boldsymbol{R}_{g}\right)\right|^{2} E_{p u}^{2}\left[\omega_{p u}-U_{e g}\left(\boldsymbol{R}_{g}\right)\right] \rho_{g}^{W i g}\left(\boldsymbol{R}_{g}, \boldsymbol{P}_{g}\right)
\end{aligned}
$$

are the quasi-classical doorway functions and

$$
\begin{aligned}
& W_{0}^{\mathrm{int}}\left(\omega_{p r}, \boldsymbol{R}_{g}(\tau), \boldsymbol{P}_{g}(\tau)\right)=\sum_{e}\left|\boldsymbol{\varepsilon}_{p r} \boldsymbol{\mu}_{g e}\left(\boldsymbol{R}_{g}(\tau)\right)\right|^{2} E_{p r}^{2}\left[\omega_{p r}-U_{e g}\left(\boldsymbol{R}_{g}(\tau)\right)\right] \\
& W_{I}^{\mathrm{int}}\left(\omega_{p r}, \boldsymbol{R}_{e}(\tau), \boldsymbol{P}_{e}(\tau)\right)=\left|\boldsymbol{\varepsilon}_{p r} \boldsymbol{\mu}_{g e(\tau)}\left(\boldsymbol{R}_{e}(\tau)\right)\right|^{2} E_{p r}^{2}\left[\omega_{p r}-U_{e(\tau) g}\left(\boldsymbol{R}_{e}(\tau)\right)\right] \\
& W_{I I}^{\mathrm{int}}\left(\omega_{p r}, \boldsymbol{R}_{e}(\tau), \boldsymbol{P}_{e}(\tau)\right)=\sum_{f}\left|\boldsymbol{\varepsilon}_{p r} \boldsymbol{\mu}_{e(\tau) f}\left(\boldsymbol{R}_{e}(\tau)\right)\right|^{2} E_{p r}^{2}\left[\omega_{p r}-U_{f e(\tau)}\left(\boldsymbol{R}_{e}(\tau)\right)\right]
\end{aligned}
$$

are the quasi-classical window functions. In the above formulas, $E_{p u}(\omega)$ and $E_{p r}(\omega)$ are the Fourier transforms of the $E_{p u}(t)$ and $E_{p r}(t)$; electronic states of the manifold $\{\mathrm{I}\}$ are denoted as $e$; electronic states of the manifold $\{$ II $\}$ are denoted as $f ; \boldsymbol{\mu}_{g e}\left(\boldsymbol{R}_{g}(\tau)\right), \boldsymbol{\mu}_{g e(\tau)}\left(\boldsymbol{R}_{e}(\tau)\right)$ and $\boldsymbol{\mu}_{e(\tau) f}\left(\boldsymbol{R}_{e}(\tau)\right)$ are the TDMs between the corresponding electronic states for a specific nuclear configuration $\boldsymbol{R}_{g}(\tau)$ or $\boldsymbol{R}_{e}(\tau) ; U_{e g}\left(\boldsymbol{R}_{e}(\tau)\right), U_{e(\tau) g}\left(\boldsymbol{R}_{e}(\tau)\right)$ and $U_{f e(\tau)}\left(\boldsymbol{R}_{e}(\tau)\right)$ are the energy differences between the corresponding electronic states for a specific nuclear configuration $\boldsymbol{R}_{g}(\tau)$ or $\boldsymbol{R}_{e}(\tau)$. The notion $e(\tau)$ means that a trajectory initiated at $t=0$ in a state $e$ of the manifold $\{$ I $\}$ can end up at time $t=\tau$ in another state of the manifold $\{$ I $\}$.

The quasi-classical formulas for the dispersed PP signal read as follows ${ }^{1}$ 


$$
\begin{aligned}
& I_{d i s}(\tau, \omega)=I_{d i s}^{G S B}(\tau, \omega)+I_{d i s}^{S E}(\tau, \omega)+I_{d i s}^{E S A}(\tau, \omega) \\
& I_{d i s}^{G S B}(\tau, \omega)=\omega_{p r} \int d \boldsymbol{R}_{g} d \boldsymbol{P}_{g} D_{0}\left(\omega_{p u}, \boldsymbol{R}_{g}, \boldsymbol{P}_{g}\right) W_{0}^{d i s}\left(\omega_{p r}, \omega, \boldsymbol{R}_{g}(\tau), \boldsymbol{P}_{g}(\tau)\right) \\
& I_{d i s}^{S E}(\tau, \omega)=\omega_{p r} \int d \boldsymbol{R}_{g} d \boldsymbol{P}_{g} D_{\mathrm{I}}\left(\omega_{p u}, \boldsymbol{R}_{g}, \boldsymbol{P}_{g}\right) W_{\mathrm{I}}^{d i s}\left(\omega_{p r}, \omega, \boldsymbol{R}_{e}(\tau), \boldsymbol{P}_{e}(\tau)\right) \\
& I_{d i s}^{E S A}(\tau, \omega)=-\omega_{p r} \int d \boldsymbol{R}_{g} d \boldsymbol{P}_{g} D_{\mathrm{I}}\left(\omega_{p u}, \boldsymbol{R}_{g}, \boldsymbol{P}_{g}\right) W_{\mathrm{II}}^{d i s}\left(\omega_{p r}, \omega, \boldsymbol{R}_{e}(\tau), \boldsymbol{P}_{e}(\tau)\right)
\end{aligned}
$$

Here the doorway functions are given by Eq. (18), while the window functions are defined as

$$
\begin{aligned}
& W_{0}^{d i s}\left(\omega_{p r}, \omega, \boldsymbol{R}_{g}(\tau), \boldsymbol{P}_{g}(\tau)\right)=E_{p r}^{2}\left[\omega-\omega_{p r}\right] \sum_{e}\left|\boldsymbol{\varepsilon}_{p r} \boldsymbol{\mu}_{g e}\left(\boldsymbol{R}_{g}(\tau)\right)\right|^{2} \frac{v}{v^{2}+\left[\omega-U_{e g}\left(\boldsymbol{R}_{g}(\tau)\right)\right]^{2}} \\
& W_{\mathrm{I}}^{d i s}\left(\omega_{p r}, \omega, \boldsymbol{R}_{e}(\tau), \boldsymbol{P}_{e}(\tau)\right)=E_{p r}^{2}\left[\omega-\omega_{p r}\right]\left|\boldsymbol{\varepsilon}_{p r} \boldsymbol{\mu}_{g e(\tau)}\left(\boldsymbol{R}_{e}(\tau)\right)\right|^{2} \frac{v}{v^{2}+\left[\omega-U_{e(\tau) g}\left(\boldsymbol{R}_{e}(\tau)\right)\right]^{2}} \\
& W_{\mathrm{II}}^{d i s}\left(\omega_{p r}, \omega, \boldsymbol{R}_{e}(\tau), \boldsymbol{P}_{e}(\tau)\right)=E_{p r}^{2}\left[\omega-\omega_{p r}\right] \sum_{f}\left|\boldsymbol{\varepsilon}_{p r} \boldsymbol{\mu}_{e(\tau) f}\left(\boldsymbol{R}_{e}(\tau)\right)\right|^{2} \frac{v}{v^{2}+\left[\omega-U_{f e(\tau)}\left(\boldsymbol{R}_{e}(\tau)\right)\right]^{2}}
\end{aligned}
$$

where $v$ is the electronic dephasing rate, which can be considered as a technical parameter of the simulation.

When the dispersed signal is detected by a spectrometer, which means it is not heterodyned with the probe pulse, we obtain $I_{d i s}(\tau, \omega) \sim \boldsymbol{P}(\tau, \omega)$. In this case, one has to replace $E_{p r}^{2}\left(\omega-\omega_{p r}\right)$ with $E_{p r}\left(\omega-\omega_{p r}\right)$ in Eq. (21). The impulsive dispersed PP signal, which has perfect time and frequency resolution, ${ }^{7}$ is obtained by setting $E_{p r}^{2}\left(\omega-\omega_{p r}\right) \rightarrow 1$ in Eq. (21).

\section{S6. Computational details}

The ground state $\left(\mathrm{S}_{0}\right)$, first excited state $\left(\mathrm{S}_{1}\right)$ and second excited state $\left(\mathrm{S}_{2}\right)$ minima of the PE dendrimer model with 2-ring and 3-ring units were optimized at DFT/CAM-B3LYP/6-31G and TDDFT/CAM-B3LYP/6-31G levels, respectively. The vibrational frequencies were calculated to ensure the optimized minima were stationary points. 
For the dynamics simulation, the initial nuclear coordinates and momenta were sampled from the Wigner distribution of the lowest vibrational level on the electronic ground state at $\mathrm{S}_{0}$-min. In this work, 200 pairs of coordinates and momenta were sampled. Based on these initial conditions, the nonadiabatic dynamics initiated at $\mathrm{S}_{1}, \mathrm{~S}_{2}$ and $\mathrm{S}_{3}$ were performed individually. The FSSH algorithm was employed to enable nonadiabatic transitions between these excited states. During the propagation of electronic wavefunction, the decoherence correction proposed by Granucci et al. was adopted, and the parameter $\alpha$ was set to $0.1{ }^{8}$ To simulate the GSB signal, the BornOppenheimer (BO) dynamics on the ground-state potential energy surface (PES) was also performed with the same initial conditions. The nuclear and electronic time steps were set to 0.5 and $0.005 \mathrm{fs}$, respectively.

In the nonadiabatic dynamics simulations, the electronic state energies, nuclear gradients, nonadiabatic coupling vectors (NACVs) and TDMs between electronics states were obtained from the on-the-fly quantum calculations in each nuclear time step.

Previous benchmark calculations of the same compound ${ }^{9}$ at several electronic-structure levels (TDDFT with different functionals, SCS-ADC(2), TDHF and DFT/MRCI) suggested that the TDDFT with CAM-B3LYP functional can well describe the excited-state properties of the current model system. Thus, the TDDFT method with CAM-B3LYP functional and 6-31G basis set was used in this work for the balance of computational efficiency and accuracy. In the TDDFT framework, analytical NACVs were directly obtained in the electronic structure calculations. ${ }^{10-11}$

To simulate the ESA signals, high-lying excited states in manifold $\{$ II $\}$ must be considered. 
Hence 146 excited states with vertical excited energies (VEEs) up to $10 \mathrm{eV}$ were incorporated in manifold $\{\mathrm{II}\}$. The VEE energies and TDMs between the electronic states of manifold $\{\mathrm{I}\}$ and those of $\{\mathrm{II}\}$ were calculated for every second time step (1 fs). 


\section{S7: Basis set benchmark}

To benchmark the results with $6-31 \mathrm{G}$ basis set, the $6-31 \mathrm{G}^{*}$ basis set added with the polarization functions was used to calculate the excited-state characters at $\mathrm{S}_{0}$-min. The excited states at $\mathrm{S}_{0}$-min were calculated at the TDDFT/CAM-B3LYP level with 6-31G and 6-31G* basis sets, as shown in Table S1. The calculations with two basis sets predicted similar VEEs and TDMs. Therefore, the 6-31G basis set is enough to describe the excited-state properties of the PE dendrimer model. In addition, when we adopt the 6-31G basis set instead of the $6-31 \mathrm{G}^{*}$ basis set in the electronic structure calculations, we can largely speed up the on-the-fly dynamics simulation and ESA signal calculations. As a result, all discussions in this work are based on the data obtained at the TDDFT/CAM-B3LYP/6-31G level.

Table S1. The VEEs, TDMs and electronic characters of the four low-lying electronic states at the TDDFT/CAM-B3LYP/6-31G level at $\mathrm{S}_{0}$-min of PE dendrimer model. The VEEs and TDMs obtained with the $6-31 \mathrm{G}^{*}$ basis set are given in parentheses.

\begin{tabular}{cccccc}
\hline & \multicolumn{3}{c}{ TEE $(\mathrm{eV})$} & \multicolumn{4}{c}{ TDM (Debye) } \\
\hline & & $\mathrm{X}$ & $\mathrm{Y}$ & $\mathrm{Z}$ & Tot \\
\hline $\mathrm{S}_{1}$ & 4.03 & 12.09 & -1.36 & 0 & 12.17 \\
& $(3.90)$ & $(12.20)$ & $(-1.40)$ & $(0)$ & $(12.28)$ \\
$\mathrm{S}_{2}$ & 4.64 & 2.88 & -5.97 & 0 & 6.63 \\
& $(4.51)$ & $(2.90)$ & $(-6.04)$ & $(0)$ & $(6.70)$ \\
$\mathrm{S}_{3}$ & 4.71 & 0.77 & -1.41 & 0 & 1.61 \\
& $(4.62)$ & $(0.56)$ & $(-0.97)$ & $(0)$ & $(1.12)$ \\
$\mathrm{S}_{4}$ & 4.99 & 0 & -0.09 & 0 & 0.09 \\
& $(4.90)$ & $(0.17)$ & $(-0.40)$ & $(0)$ & $(0.43)$ \\
\hline
\end{tabular}




\section{S8: The VEEs and values of key DoFs at key geometries.}

The VEEs and the key DoFs at $\mathrm{S}_{0}$-min, $\mathrm{S}_{1}$-min and $\mathrm{S}_{2}$-min, including the lengths of the three ethynylene bonds $\left(r_{1}, r_{2}\right.$ and $\left.r_{3}\right)$, are presented in Table $S 2$. At $S_{0}-m i n$, three CC triple bonds $\left(r_{1}, r_{2}\right.$ and $r_{3}$ ) display the same lengths. At $S_{1}-\min , r_{2}$ and $r_{3}$ increase by $0.023 \AA$, while at $S_{2}-m i n, r_{1}$ increases by $0.037 \AA$. Therefore, the former two and the later one stretching motions show significant vibronic couplings for $S_{1}$ and $S_{2}$, respectively.

Table S2. The VEEs and values of key DoFs at $S_{0}-\min , S_{1}-\min$ and $S_{2}$-min.

\begin{tabular}{llllllll}
\hline Geometry & $\mathrm{S}_{1}(\mathrm{eV})$ & $\mathrm{S}_{2}(\mathrm{eV})$ & $\mathrm{S}_{3}(\mathrm{eV})$ & $\mathrm{S}_{4}(\mathrm{eV})$ & $\mathrm{r}_{1}(\AA)$ & $\mathrm{r}_{2}(\AA)$ & $\mathrm{r}_{3}(\AA)$ \\
\hline $\mathrm{S}_{0}$-min & 4.03 & 4.64 & 4.71 & 4.99 & 1.213 & 1.213 & 1.213 \\
$\mathrm{~S}_{1}$-min & 3.50 & 4.50 & 4.62 & 4.71 & 1.213 & 1.236 & 1.236 \\
$\mathrm{~S}_{2}$-min & 3.92 & 4.13 & 4.45 & 4.91 & 1.250 & 1.217 & 1.216 \\
\hline
\end{tabular}




\section{S9: Frontier molecular orbitals at $\mathbf{S}_{0}-\mathbf{m i n}$}
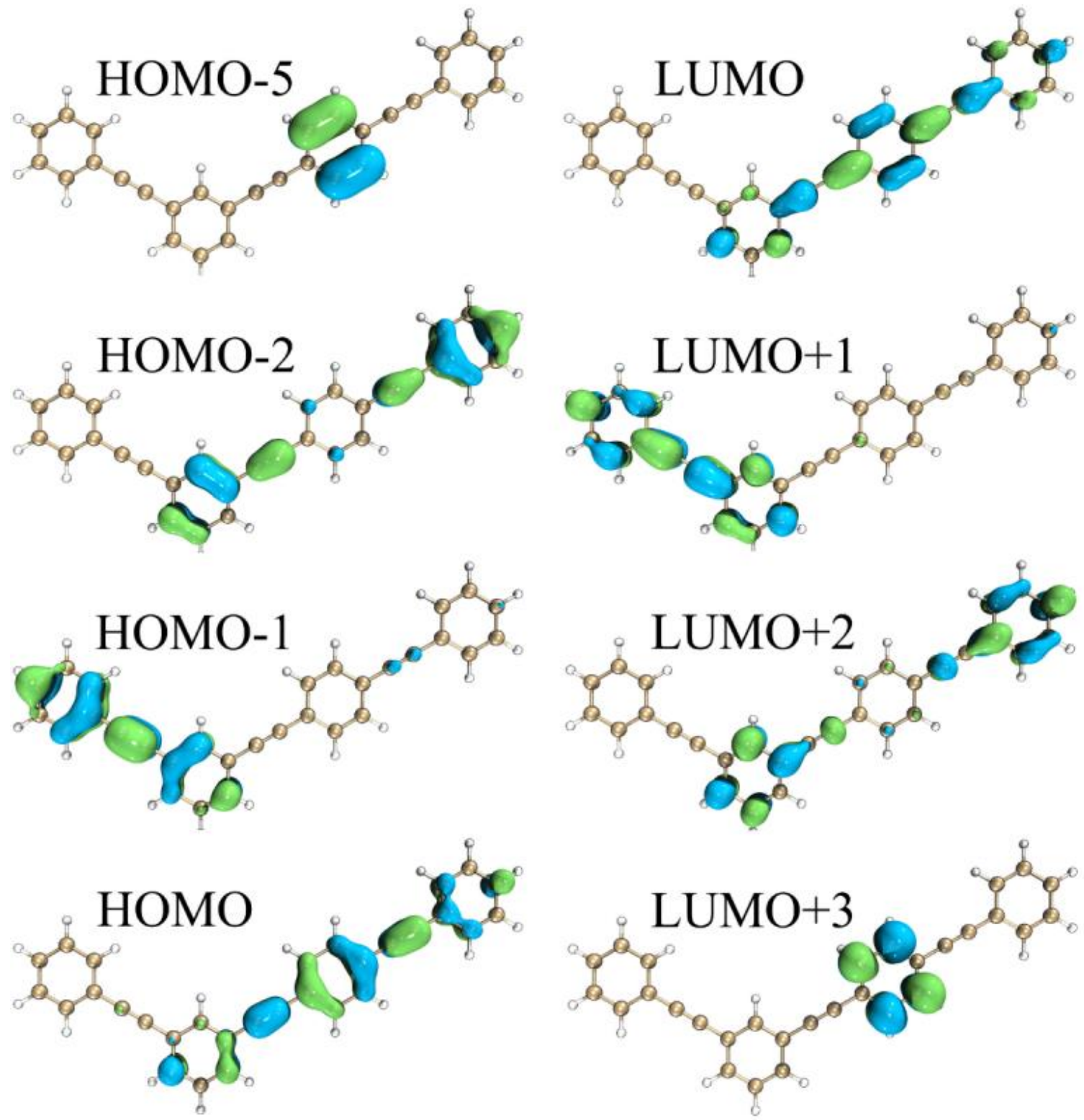

Figure S1. Frontier molecular orbitals at $\mathrm{S}_{0}$-min of PE dendrimer model. 


\section{S10: The vertical excitation energies and transition dipole moments along the stretching of $\mathrm{CC}$ triple bonds.}

The VEEs and TDM X/Y components for the first three excited states along the stretching of the CC triple bonds $\left(\mathrm{r}_{1}, \mathrm{r}_{2}, \mathrm{r}_{3}\right)$ are shown in Figure $\mathrm{S} 2$. The VEE of $\mathrm{S}_{1}$ is sensitive to the stretching of $r_{2}$ and $r_{3}$, while the VEEs of $S_{2}$ are sensitive to the stretching of $r_{1}$. The TDM of $S_{1}$ is only sensitive to the stretching of $r_{1}$. The changes of the TDMs of $S_{2}$ and $S_{3}$ are much more complicated due to the flips of the electronic states with the stretching of the $\mathrm{CC}$ triple bonds. In addition, we also noticed that the $S_{1}$ and $S_{2}$ states are close to each other at $r_{1} \sim 1.34 \AA$. This indicates that the $r_{1}$ elongation induces the $S_{2}$ to $S_{1}$ nonadiabatic decay. 

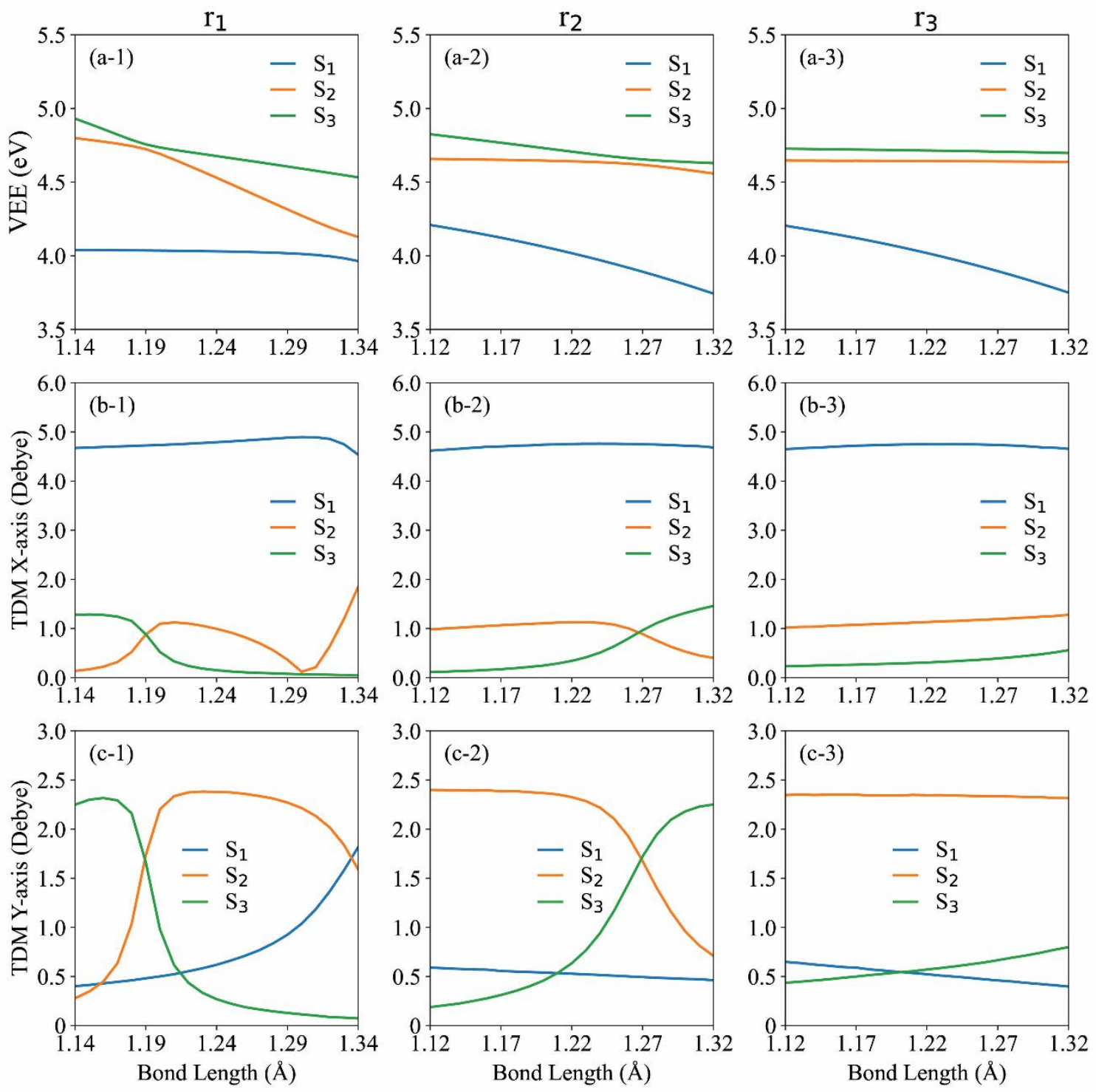

Figure S2. (a) vertical excitation energies (VEEs) as well as absolute values of transition dipole moment (TDM) components in the X-axis (b) and Y-axis (c) direction along (1) $r_{1}$, (2) $r_{2}$ and (3) $\mathrm{r}_{3} \mathrm{CC}$ triple stretches for the first three excited states $\mathrm{S}_{1}, \mathrm{~S}_{2}$ and $\mathrm{S}_{3}$. 


\section{S11: Time-dependent distributions of $\mathrm{CC}$ triple bonds.}

The time-dependent distributions of the CC triple bonds $\left(r_{1}, r_{2}, r_{3}\right)$, as well as their symmetric and antisymmetric combinations, for the dynamic simulations initiated from different electronic states are shown in Figure S3. When the BO dynamics on the ground state was performed, as shown in the left panels in Figure S3, no obvious CC stretching motions is observed. When the dynamics started from $S_{1}$, as shown in the middle panels in Figure $S 3$, the significant stretching motions of $r_{2}$ and $r_{3}$, and particularly of their symmetric combination, are observed. When the nonadiabatic dynamics started from $\mathrm{S}_{2}$, as shown in the right panels in Figure $\mathrm{S} 3$, the significant stretching motions of $r_{1}$ are observed at the beginning of the dynamics. Then the stretching motion of $r_{1}$ becomes weak while the stretching motion of $r_{2}$ becomes strong. This indicates that the $S_{2}$ to $\mathrm{S}_{1}$ nonadiabatic decay takes place during the dynamics evolution. 

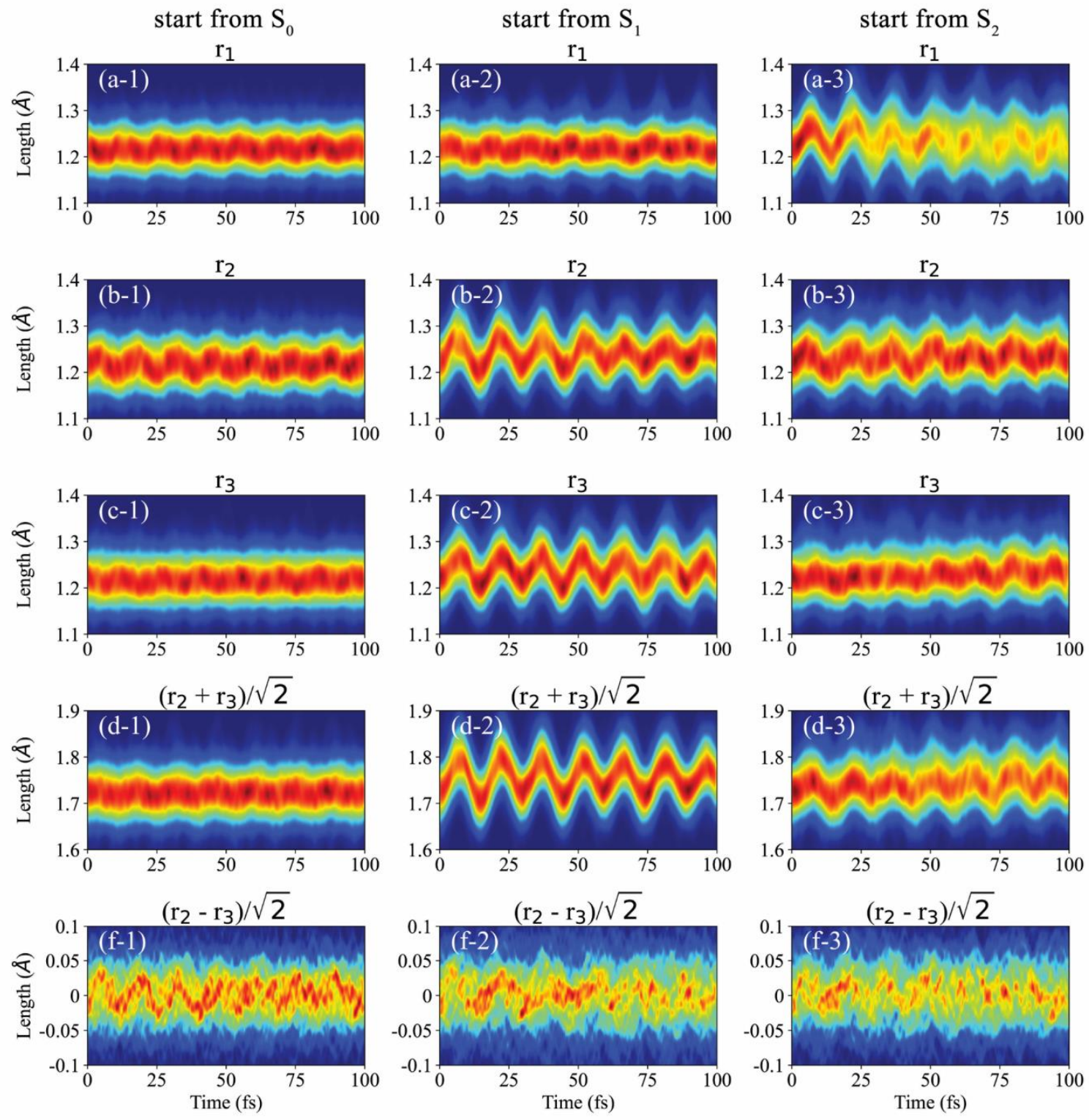

Figure S3. The time-dependent length distributions of $r_{1}, r_{2}, r_{3},\left(r_{2}+r_{3}\right) / \sqrt{2}$ and $\left(r_{2}-r_{3}\right) / \sqrt{2}$ of PE dendrimer model for the dynamics initiated at $S_{0}$ (left column), $S_{1}$ (middle column) and $S_{2}$ (right column). 200 trajectories were used for all dynamics. 


\section{S12: TA PP dispersed signals.}

The TA PP dispersed signals of the PE dendrimer model with the pump pulses centered at $\omega_{p u}=4.03 \mathrm{eV}$ and $\omega_{p u}=4.64 \mathrm{eV}$ are shown in Figure S4 and Figure S5, respectively. In the simulations, the dephasing parameter for the dispersed signal was chosen as $v=0.02 \mathrm{eV}$. The white light detection was assumed in the simulation so that the TA PP signal $I_{d i s}(\tau, \omega)$ covers the entire spectral range of interest, from $0.5 \mathrm{eV}$ to $5.5 \mathrm{eV}$. Although the dispersed signals are more diffuse than the integral signals, all features of the dispersed GSB, SE and ESA signals (in Figures S3 and S4), including the oscillation patterns, spectral shifts, rise and decay times, are very similar to their integral counterparts in Figures 3 and 4. 

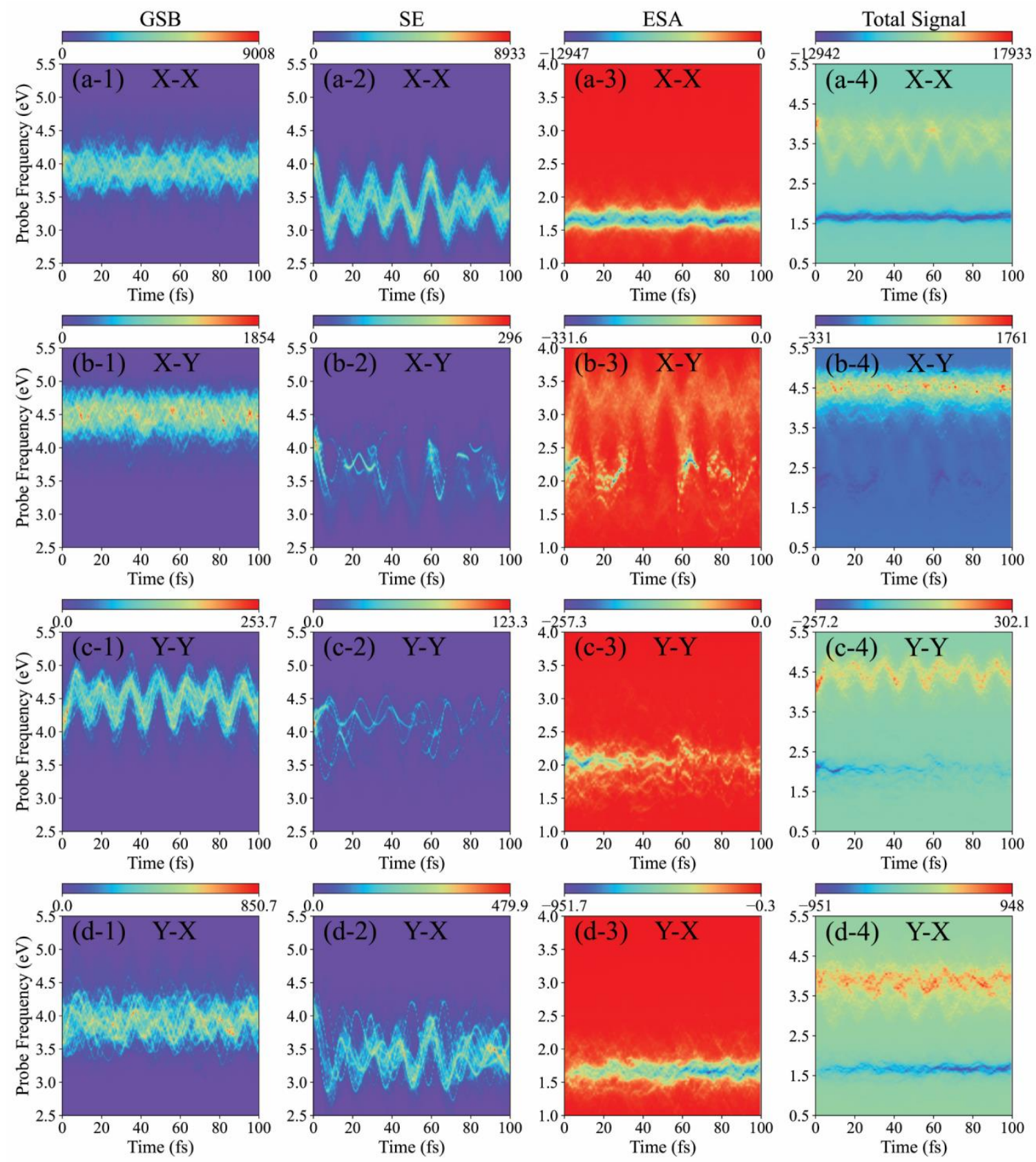

Figure S4. (1) GSB, (2) SE, (3) ESA contributions and (4) total dispersed signal of $I_{d i s}(\tau, \omega)$ as a function of $\tau$ and $\omega$. The notation $\alpha-\beta(\alpha, \beta=\mathrm{X}, \mathrm{Y})$ in each panel indicates that the pump pulse polarization $\varepsilon_{p u}$ is along the $\alpha$-axis direction, while the probe pulse polarization $\varepsilon_{p r}$ is along the $\beta$-axis direction. The pump pulse with $\omega_{p u}=4.03 \mathrm{eV}$ is used. 

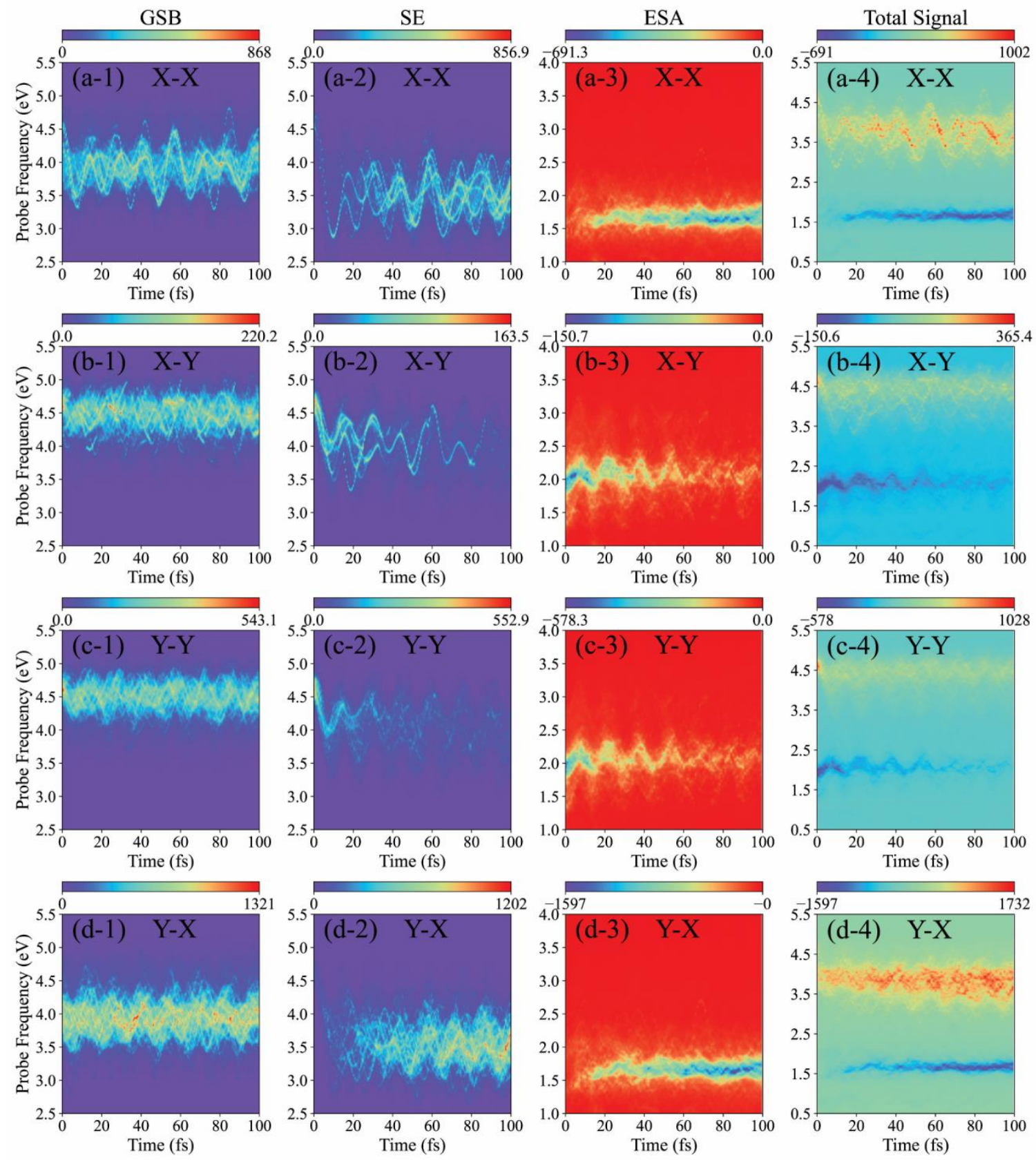

Figure S5. (1) GSB, (2) SE, (3) ESA contributions and (4) total dispersed signal of $I_{d i s}(\tau, \omega)$ as a function of $\tau$ and $\omega$. The notation $\alpha-\beta(\alpha, \beta=\mathrm{X}, \mathrm{Y})$ in each panel indicates that the pump pulse polarization $\boldsymbol{\varepsilon}_{p u}$ is along the $\alpha$-axis direction, while the probe pulse polarization $\boldsymbol{\varepsilon}_{p r}$ is along the $\beta$-axis direction. The pump pulse with $\omega_{p u}=4.64 \mathrm{eV}$ is used. 


\section{S13: Long-time TA PP integral signals}

The long-time (200 fs) TA PP integral signals of the PE dendrimer model with the pump pulses centered at $\omega_{p u}=4.03 \mathrm{eV}$ and $\omega_{p u}=4.64 \mathrm{eV}$ are shown in Figure S6 and Figure S7, respectively. The main features of the TA PP signals with 200 fs simulation time (Figure S6 and Figure S7) are very similar to those of the signals with 100 fs simulation time (Figure 3 and Figure 4). This indicates that no quenching processes occur on the timescale of at least $200 \mathrm{fs}$. 

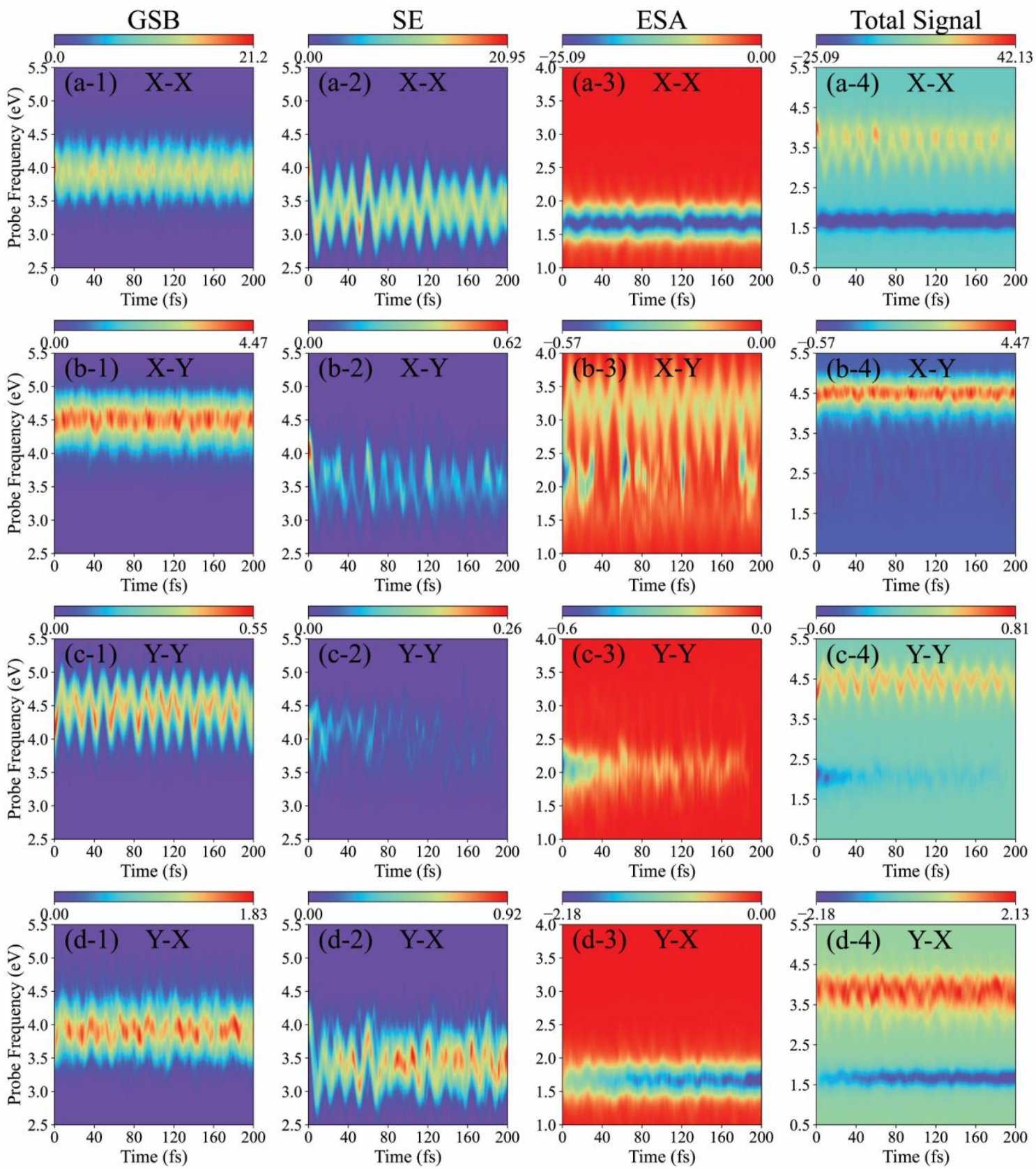

Figure S6. (1) GSB, (2) SE, (3) ESA contributions and (4) total integral signal of $I_{\text {int }}\left(\tau, \omega_{p r}\right)$ as a function of $\tau$ and $\omega_{p r}$. The notation $\alpha-\beta(\alpha, \beta=\mathrm{X}, \mathrm{Y})$ in each panel indicates that the pump pulse polarization $\boldsymbol{\varepsilon}_{p u}$ is along the $\alpha$-axis direction, while the probe pulse polarization $\boldsymbol{\varepsilon}_{p r}$ is along the $\beta$-axis direction. The pump pulse with $\omega_{p u}=4.03 \mathrm{eV}$ is used. The simulation time is $200 \mathrm{fs}$. 

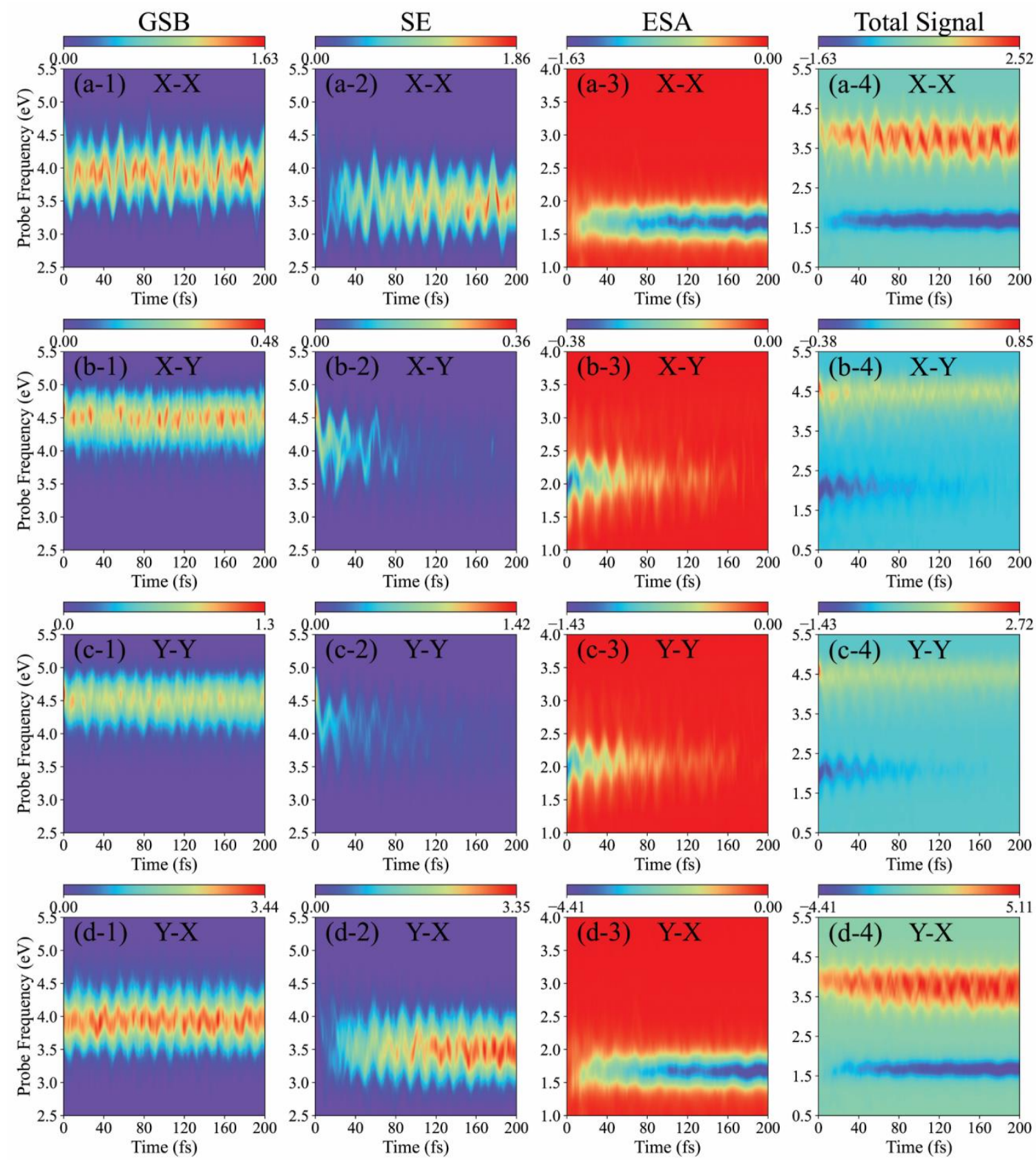

Figure S7. (1) GSB, (2) SE, (3) ESA contributions and (4) total integral signal of $I_{\text {int }}\left(\tau, \omega_{p r}\right)$ as a function of $\tau$ and $\omega_{p r}$. The notation $\alpha-\beta(\alpha, \beta=\mathrm{X}, \mathrm{Y})$ in each panel indicates that the pump pulse polarization $\varepsilon_{p u}$ is along the $\alpha$-axis direction, while the probe pulse polarization $\varepsilon_{p r}$ is along the $\beta$-axis direction. The pump pulse with $\omega_{p u}=4.64 \mathrm{eV}$ is used. The simulation time is $200 \mathrm{fs}$. 


\section{References}

(1) Gelin, M. F.; Huang, X.; Xie, W.; Chen, L.; Doslic, N. A.; Domcke, W. Ab initio surfacehopping simulation of femtosecond transient-absorption pump-probe signals of nonadiabatic excited-state dynamics using the doorway-window representation. J. Chem. Theory Comput. 2021, 17, 2394-2408.

(2) Mukamel, S. Principles of nonlinear optical spectroscopy. 1995.

(3) Ren, H.; Fingerhut, B. P.; Mukamel, S. Time resolved photoelectron spectroscopy of thioflavin T photoisomerization: A simulation study. J. Phys. Chem. A 2013, 117, 6096-6104.

(4) Yan, Y. J.; Mukamel, S. Femtosecond pump-probe spectroscopy of polyatomic-molecules in condensed phases. Phys. Rev. A 1990, 41, 6485-6504.

(5) Yan, Y. J.; Fried, L. E.; Mukamel, S. Ultrafast pump-probe spectroscopy - femtosecond dynamics in Liouville space. J. Phys. Chem. 1989, 93, 8149-8162.

(6) Fried, L. E.; Mukamel, S. A classical-theory of pump-probe photodissociation for arbitrary pulse durations. J. Chem. Phys. 1990, 93, 3063-3071.

(7) Palacino-González, E.; Gelin, M. F.; Domcke, W. Analysis of transient-absorption pump-probe signals of nonadiabatic dissipative systems: "Ideal" and "real" spectra. J. Chem. Phys. 2019, 150, 204102.

(8) Granucci, G.; Persico, M. Critical appraisal of the fewest switches algorithm for surface hopping. J. Chem. Phys. 2007, 126, 134114.

(9) Huang, J.; Du, L.; Hu, D.; Lan, Z. Theoretical analysis of excited states and energy transfer mechanism in conjugated dendrimers. J. Comput. Chem. 2015, 36, 151-163.

(10) Zhang, X.; Herbert, J. M. Analytic derivative couplings for spin-flip configuration interaction singles and spin-flip time-dependent density functional theory. J. Chem. Phys. 2014, 141, 064104. (11) Ou, Q.; Fatehi, S.; Alguire, E.; Shao, Y.; Subotnik, J. E. Derivative couplings between TDDFT excited states obtained by direct differentiation in the Tamm-Dancoff approximation. $J$. Chem. Phys. 2014, 141, 024114 
(12) Hu, D.; Liu, Y. F.; Sobolewski, A. L.; Lan, Z. Nonadiabatic dynamics simulation of keto isocytosine: a comparison of dynamical performance of different electronic-structure methods. Phys. Chem. Chem. Phys. 2017, 19, 19168-19177.

(13) Du, L. K.; Lan, Z. G. An on-the-fly surface-hopping program JADE for nonadiabatic molecular dynamics of polyatomic systems: Implementation and applications. J. Chem. Theory Comput. 2015, 11, 1360-1374.

(14) Frisch, M. J.; Trucks, G. W.; Schlegel, H. B.; Scuseria, G. E.; Robb, M. A.; Cheeseman, J. R.; Scalmani, G.; Barone, V.; Petersson, G. A.; Nakatsuji, H.; Li, X.; Caricato, M.; Marenich, A. V.; Bloino, J.; Janesko, B. G.; Gomperts, R.; Mennucci, B.; Hratchian, H. P.; Ortiz, J. V.; Izmaylov, A. F.; Sonnenberg, J. L.; Williams; Ding, F.; Lipparini, F.; Egidi, F.; Goings, J.; Peng, B.; Petrone, A.; Henderson, T.; Ranasinghe, D.; Zakrzewski, V. G.; Gao, J.; Rega, N.; Zheng, G.; Liang, W.; Hada, M.; Ehara, M.; Toyota, K.; Fukuda, R.; Hasegawa, J.; Ishida, M.; Nakajima, T.; Honda, Y.; Kitao, O.; Nakai, H.; Vreven, T.; Throssell, K.; Montgomery Jr., J. A.; Peralta, J. E.; Ogliaro, F.; Bearpark, M. J.; Heyd, J. J.; Brothers, E. N.; Kudin, K. N.; Staroverov, V. N.; Keith, T. A.; Kobayashi, R.; Normand, J.; Raghavachari, K.; Rendell, A. P.; Burant, J. C.; Iyengar, S. S.; Tomasi, J.; Cossi, M.; Millam, J. M.; Klene, M.; Adamo, C.; Cammi, R.; Ochterski, J. W.; Martin, R. L.; Morokuma, K.; Farkas, O.; Foresman, J. B.; Fox, D. J. Gaussian 16 Rev. C.01, Wallingford, CT, 2016.

(15) Shao, Y.; Gan, Z.; Epifanovsky, E.; Gilbert, A. T. B.; Wormit, M.; Kussmann, J.; Lange, A. W.; Behn, A.; Deng, J.; Feng, X.; Ghosh, D.; Goldey, M.; Horn, P. R.; Jacobson, L. D.; Kaliman, I.; Khaliullin, R. Z.; Kus, T.; Landau, A.; Liu, J.; Proynov, E. I.; Rhee, Y. M.; Richard, R. M.; Rohrdanz, M. A.; Steele, R. P.; Sundstrom, E. J.; Woodcock, H. L., III; Zimmerman, P. M.; Zuev, D.; Albrecht, B.; Alguire, E.; Austin, B.; Beran, G. J. O.; Bernard, Y. A.; Berquist, E.; Brandhorst, K.; Bravaya, K. B.; Brown, S. T.; Casanova, D.; Chang, C.-M.; Chen, Y.; Chien, S. H.; Closser, K. D.; Crittenden, D. L.; Diedenhofen, M.; DiStasio, R. A., Jr.; Do, H.; Dutoi, A. D.; Edgar, R. G.; Fatehi, S.; Fusti-Molnar, L.; Ghysels, A.; Golubeva-Zadorozhnaya, A.; Gomes, J.; Hanson-Heine, 
M. W. D.; Harbach, P. H. P.; Hauser, A. W.; Hohenstein, E. G.; Holden, Z. C.; Jagau, T.-C.; Ji, H.; Kaduk, B.; Khistyaev, K.; Kim, J.; Kim, J.; King, R. A.; Klunzinger, P.; Kosenkov, D.; Kowalczyk, T.; Krauter, C. M.; Lao, K. U.; Laurent, A. D.; Lawler, K. V.; Levchenko, S. V.; Lin, C. Y.; Liu, F.; Livshits, E.; Lochan, R. C.; Luenser, A.; Manohar, P.; Manzer, S. F.; Mao, S.-P.; Mardirossian, N.; Marenich, A. V.; Maurer, S. A.; Mayhall, N. J.; Neuscamman, E.; Oana, C. M.; Olivares-Amaya, R.; O'Neill, D. P.; Parkhill, J. A.; Perrine, T. M.; Peverati, R.; Prociuk, A.; Rehn, D. R.; Rosta, E.; Russ, N. J.; Sharada, S. M.; Sharma, S.; Small, D. W.; Sodt, A.; Stein, T.; Stueck, D.; Su, Y.-C.; Thom, A. J. W.; Tsuchimochi, T.; Vanovschi, V.; Vogt, L.; Vydrov, O.; Wang, T.; Watson, M. A.; Wenzel, J.; White, A.; Williams, C. F.; Yang, J.; Yeganeh, S.; Yost, S. R.; You, Z.-Q.; Zhang, I. Y.; Zhang, X.; Zhao, Y.; Brooks, B. R.; Chan, G. K. L.; Chipman, D. M.; Cramer, C. J.; Goddard, W. A., III; Gordon, M. S.; Hehre, W. J.; Klamt, A.; Schaefer, H. F., III; Schmidt, M. W.; Sherrill, C. D.; Truhlar, D. G.; Warshel, A.; Xu, X.; Aspuru-Guzik, A.; Baer, R.; Bell, A. T.; Besley, N. A.; Chai, J.-D.; Dreuw, A.; Dunietz, B. D.; Furlani, T. R.; Gwaltney, S. R.; Hsu, C.P.; Jung, Y.; Kong, J.; Lambrecht, D. S.; Liang, W.; Ochsenfeld, C.; Rassolov, V. A.; Slipchenko, L. V.; Subotnik, J. E.; Van Voorhis, T.; Herbert, J. M.; Krylov, A. I.; Gill, P. M. W.; Head-Gordon, M. Advances in molecular quantum chemistry contained in the Q-Chem 4 program package. Mol. Phys. 2015, 113, 184-215. 